\title{
GaAs/Ge crystals grown on Si substrates patterned down to the micron scale
}

Cite as: J. Appl. Phys. 119, 055301 (2016); https://doi.org/10.1063/1.4940379

Submitted: 08 September 2015 . Accepted: 09 January 2016 . Published Online: 02 February 2016

A. G. Taboada, M. Meduňa, M. Salvalaglio, F. Isa, T. Kreiliger, C. V. Falub, E. Barthazy Meier, E. Müller, L. Miglio, G. Isella, and H. von Känel

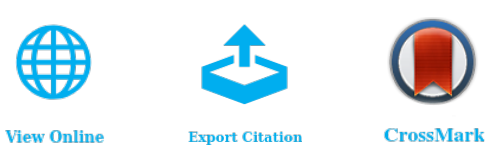

\section{ARTICLES YOU MAY BE INTERESTED IN}

GaAs/Ge/Si epitaxial substrates: Development and characteristics

AIP Advances 7, 015304 (2017); https://doi.org/10.1063/1.4974498

Strain relaxation of GaAs/Ge crystals on patterned Si substrates

Applied Physics Letters 104, 022112 (2014); https://doi.org/10.1063/1.4861864

Kinetic growth mode of epitaxial GaAs on $\mathrm{Si}(001)$ micro-pillars

Journal of Applied Physics 120, 245702 (2016); https://doi.org/10.1063/1.4972467

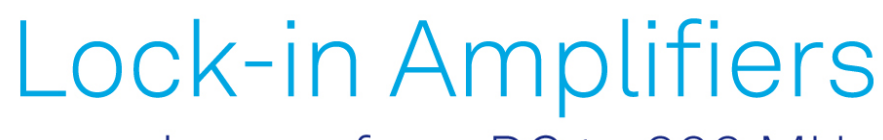

... and more, from DC to $600 \mathrm{MHz}$

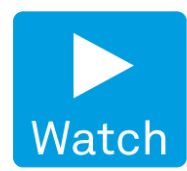




\title{
GaAs/Ge crystals grown on Si substrates patterned down to the micron scale
}

\author{
A. G. Taboada, ${ }^{1, a)}$ M. Meduña, ${ }^{2,3}$ M. Salvalaglio, ${ }^{4}$ F. Isa, ${ }^{1,5}$ T. Kreiliger, ${ }^{1}$ C. V. Falub, ${ }^{1, b)}$ \\ E. Barthazy Meier, ${ }^{6}$ E. Müller, ${ }^{6}$ L. Miglio, ${ }^{4}$ G. Isella, ${ }^{5}$ and H. von Känel ${ }^{1}$ \\ ${ }_{1}^{1}$ Laboratory for Solid State Physics, ETH Zürich, Otto-Stern-Weg. 1, CH-8093 Zürich, Switzerland \\ ${ }^{2}$ Department of Condensed Matter Physics, Masaryk University, Kotlářská 2, CZ-61137 Brno, Czech Republic \\ ${ }^{3}$ CEITEC, Masaryk University Kamenice 5, CZ-60177 Brno, Czech Republic \\ ${ }^{4}$ L-NESS, Department of Materials Science, Università di Milano-Bicocca, Via R. Cozzi 55, I-20125 Milano, \\ Italy \\ ${ }^{5}$ L-NESS and Department of Physics, Politecnico di Milano, via Anzani 42, I-22100 Como, Italy \\ ${ }^{6}$ Scientific Center for Optical and Electron Microscopy (ScopeM), ETH Zürich, Auguste-Piccard-Hof 1, \\ CH-8093 Zürich, Switzerland
}

(Received 8 September 2015; accepted 9 January 2016; published online 2 February 2016)

\begin{abstract}
Monolithic integration of III-V compounds into high density Si integrated circuits is a key technological challenge for the next generation of optoelectronic devices. In this work, we report on the metal organic vapor phase epitaxy growth of strain-free GaAs crystals on Si substrates patterned down to the micron scale. The differences in thermal expansion coefficient and lattice parameter are adapted by a $2-\mu \mathrm{m}$-thick intermediate Ge layer grown by low-energy plasma enhanced chemical vapor deposition. The GaAs crystals evolve during growth towards a pyramidal shape, with lateral facets composed of $\{111\}$ planes and an apex formed by $\{137\}$ and (001) surfaces. The influence of the anisotropic GaAs growth kinetics on the final morphology is highlighted by means of scanning and transmission electron microscopy measurements. The effect of the Si pattern geometry, substrate orientation, and crystal aspect ratio on the GaAs structural properties was investigated by means of high resolution X-ray diffraction. The thermal strain relaxation process of GaAs crystals with different aspect ratio is discussed within the framework of linear elasticity theory by Finite Element Method simulations based on realistic geometries extracted from cross-sectional scanning electron microscopy images. (C) 2016 AIP Publishing LLC.
\end{abstract}

[http://dx.doi.org/10.1063/1.4940379]

\section{INTRODUCTION}

On-chip integration of optical and electrical functionalities will have a strong impact on the semiconductor industry in the near-future. ${ }^{1}$ In spite of its high thermal conductivity, mechanical robustness, and stable oxide, the use of $\mathrm{Si}$ is limited for optoelectronic applications due to its indirect bandgap. Conversely, the band structure of most III-V materials, and especially of GaAs, makes them particularly convenient for the design and fabrication of optoelectronic devices. In order to manufacture device quality GaAs monolithically integrated on Si significant drawbacks need to be overcome, such as the elimination of defects caused by the mismatch of lattice parameters and thermal expansion coefficients $(4.1 \%$ and $120.4 \%$, respectively, at $300 \mathrm{~K})$.

Anti-phase domains (APD) related disorder is another usual problem when growing a polar compound (e.g., GaAs) on a non-polar substrate (like $\mathrm{Si}$ or Ge). Due to the lower symmetry of the GaAs zinc-bend structure compared with the diamond cubic structure characteristic of $\mathrm{Si}$ and $\mathrm{Ge}$, two orthogonal GaAs sublattices may independently nucleate on surface terraces separated by a single atomic step of the $\mathrm{Si}$ substrate. This leads to the formation of Ga-Ga and As-As

\footnotetext{
a) Author to whom correspondence should be addressed. Electronic mail: gonzalez@phys.ethz.ch. Present address: Camlin Technologies CH, Technoparkstrasse 1, 8005 Zürich, Switzerland.

b) Present address: Evatec AG, Hauptstrasse 1a, CH-9477 Trübbach, Switzerland.
}

bonds at the boundaries between neighboring domains, which provide deep intra-gap energy levels that act as scattering and recombination centers. ${ }^{2}$ The formation of APD can be avoided by the use of offcut substrates annealed at high temperature, which yields surfaces with a larger density of atomic double steps. ${ }^{3}$

The monolithic integration of GaAs on Si substrates has attracted the interest of numerous research groups in the past decades. Diverse approaches to the problem were attempted, such as low temperature nucleation by both metal organic vapor phase epitaxy (MOVPE) $)^{4-6}$ and molecular beam epitaxy (MBE). ${ }^{7-9}$ Several attempts to reduce the high threading dislocation density (TDD) resulting from the direct growth of $\mathrm{GaAs}$ on $\mathrm{Si}$ involve the growth of graded $\mathrm{InGaP}$ and GaAsP intermediate layers. ${ }^{10,11}$ An extension of this method consists of the integration of GaP-GaAs or InGaAs/GaAs strained super-lattices as transition layers which actively deflect the threading dislocations. ${ }^{12-14}$ Probably the most widespread procedure involves the integration of $\mathrm{Ge}$ and SiGe virtual substrates. ${ }^{15-18}$ This method is particularly interesting due to the similarities in lattice parameter $(0.08 \%$ mismatch) and thermal expansion coefficient (15\% mismatch) between GaAs and Ge. ${ }^{19-21}$

Our approach consists of applying the concept of threedimensional (3D) hetero-epitaxy to the integration of III-V materials with the mainstream Si technology. ${ }^{22}$ Undesired consequences of the thermal expansion coefficient mismatch between GaAs and $\mathrm{Si}$ such as wafer bowing and crack 
formation are avoided altogether by the decoupled growth of the III-V compound on arrays of $\mathrm{Ge} / \mathrm{Si}$ micrometer $(\mu \mathrm{m})$ sized virtual substrates. Furthermore, if the crystals exhibit sufficiently large aspect ratios threading dislocations can escape laterally at the crystal sidewalls by the so-called aspect ratio trapping (ART) mechanism. ${ }^{23,24}$ The facet formation during the crystal growth, together with the previously mentioned ART mechanism, plays an important role in the TDD reduction. Dislocations glide perpendicular to the growth front and a fraction of them are expelled through the facets while the crystal height increases. The 3Dheteroepitaxy concept has been applied to the growth of various materials (e.g., $\mathrm{Si}, \mathrm{SiGe}, \mathrm{SiC}$, and $\mathrm{GaAs}$ ) as well as more complex heterostructures (e.g., SiGe/Si and $\mathrm{InGaAs} / \mathrm{GaAs}$ QWs) on top of patterned Si substrates. ${ }^{25-29}$

In this report, we present a detailed morphological and structural characterization of $\mathrm{GaAs} / \mathrm{Ge}$ crystals grown on patterned Si substrates by MOVPE. We will first describe the characteristics of the Ge virtual $\mu \mathrm{m}$-substrates employed to adapt the GaAs crystals to the patterned $\mathrm{Si}$ substrates. Then, the distinctive kinetics of the GaAs growth on $\mathrm{Ge} / \mathrm{Si}$ patterns will be analyzed and related with the final crystal morphology. Finally, we will discuss the strain relaxation process of the GaAs/Ge crystals and its dependence on the GaAs crystal thickness, the $\mu \mathrm{m}$-substrate size and its orientation.

\section{METHOD}

Offcut ( $6^{\circ}$ towards [110]) and on-axis $\mathrm{Si}(001)$ substrates were patterned by conventional photolithography and deep reactive ion etching (DRIE) based on the Bosch process. ${ }^{30}$ The patterns consisted of $8-\mu \mathrm{m}$ tall pillars, spaced uniformly by gaps of 3 and $4 \mu \mathrm{m}$. The Si pillar width ranged from 2 to $40 \mu \mathrm{m}$. Patterns including other geometries like Si ridges $10 \mu \mathrm{m}$ in width or Si rectangles $(30 \mu \mathrm{m} \times 15 \mu \mathrm{m})$ were studied as well. Two micrometer tall Ge mesas were first grown by low energy plasma enhanced chemical vapor deposition (LEPECVD) at $\mathrm{T}=500^{\circ} \mathrm{C}$ and a growth rate of $4.2 \mathrm{~nm} / \mathrm{s}$ and cyclically annealed between 600 and $780^{\circ} \mathrm{C}$. $^{31}$ The widely used two-step method was employed for the GaAs growth by MOVPE. The $\mathrm{Ge} / \mathrm{Si}$ substrates were first annealed up to $700{ }^{\circ} \mathrm{C}$ in $\mathrm{N}_{2}$, in order to increase the density of double atomic steps. Then, a self-limited As monolayer was deposited by exposing the Ge surface to $\mathrm{AsH}_{3}$ at $\mathrm{T}=500^{\circ} \mathrm{C}$ followed by a $7 \mathrm{~nm}$ thick GaAs seed layer deposited at the same temperature. GaAs crystals with heights between 2 and $6 \mu \mathrm{m}$ were grown onto the $\mathrm{Ge} / \mathrm{Si}$ mesas at $\mathrm{T}=680^{\circ} \mathrm{C}$ and a growth rate of $28 \mathrm{~nm} / \mathrm{min}$. GaAs layers grown on offcut planar $\mathrm{Ge} / \mathrm{Si}$ substrates served as control samples. The morphology of the $\mathrm{GaAs} / \mathrm{Ge} / \mathrm{Si}$ crystals was characterized with a Zeiss ULTRA 55 scanning electron microscope (SEM). The crystal facets were identified by AFM and SEM inspection. Stereographic maps are obtained from AFM images and the results compared with data already published in the literature. ${ }^{32,33}$ Cross sections of the crystals were obtained by focused ion beam (FIB) milling in a Zeiss NVision 40 dual beam FIB/SEM system.
The structural properties of the GaAs/Ge crystals were evaluated by high resolution X-ray diffraction (HRXRD) performed with $\mathrm{Cu} \mathrm{K} \alpha 1$ radiation using two diffractometers, a PANalytical X'Pert Pro-MRD and a Rigaku SmartLab rotating anode diffractometer both equipped with a 4-bounce $\mathrm{Ge}(220)$ crystal monochromator on the incident beam, and an analyzer crystal in front of a point detector on the diffracted beam. The structural characterization was completed with transmission electron microscopy (TEM) measurements performed with a Tecnai F30ST transmission electron microscope (FEI), operated at $300 \mathrm{kV}$. The specimens for TEM investigations were thinned to electron transparency by mechanical thinning followed by Ar-ion milling (acceleration voltage $4.3 \mathrm{kV}$, etching angle $4^{\circ}$, final polishing at $1 \mathrm{kV}$ acceleration voltage). The TDD of the Ge crystals was evaluated using the etch pit count technique after wet etching in an iodine solution. ${ }^{34}$ Thereafter, etch pits formed at the emerging TDs were counted after imaging the semiconductor surface by atomic force microscopy, SEM, and Nomarski microscopy.

Elasticity-theory calculations by Finite Element Method (FEM) simulations were performed to assess the effect of patterning on the relaxation of thermal deformations. The thermal strain $\epsilon_{\text {th }}$, originating from the difference in the thermal expansion coefficients (of GaAs and Ge with respect to $\mathrm{Si}$ ), is imposed as hydrostatic strain $\epsilon_{i j}=\epsilon_{\mathrm{th}} \delta_{i j}$. The exact elastic field in the structure is then computed by the FEM simulations to satisfy both the elastic equilibrium condition with no external forces, $\nabla \cdot \sigma=0$, and the boundary condition at the free surfaces $\sigma \cdot \hat{n}=0$, where $\sigma$ is the stress tensor obtained by multiplying $\epsilon$ by the stiffness tensor $\mathrm{C}$, here considered to be anisotropic. ${ }^{35}$ The commercial FEM package COMSOL Multiphysics was used. Full 3D calculations were carried out and the experimental geometries were approximated by 3D-crystals with $\mathrm{C} 4$ symmetry around the vertical axis, based on top- and cross-sectional SEM images of the (1-10) plane. The modeled structures allow simulating a GaAs crystal of thickness ranging between 2 and $6 \mu \mathrm{m}$ grown on a $\mathrm{Si}$ pillar of widths varying between 2 and $15 \mu \mathrm{m}$. The Si pillar height assumed for the calculations is $7 \mu \mathrm{m}$, in agreement with an average of different experimental shapes. No effect of the Si substrate offcut was accounted for in these simulations. Control simulations performed to evaluate the error introduced while neglecting the effect of the misoriented substrate result in relative errors below $1 \%$ in the average strain. A large Si domain (parallelepiped in shape, more than five times thicker higher, and wider than a Si pillar) with rigid bottom and gliding boundary conditions for the lateral sidewalls was placed under the pillars mimicking an infinite substrate. A detailed summary of the different structures analyzed during this work can be found in Table I.

\section{RESULTS AND DISCUSSION}

The intermediate steps of the GaAs/Ge/Si structure fabrication are summarized on the perspective SEM micrographs shown in Figures 1(a)-1(c) for the particular case of $2-\mu \mathrm{m}$-wide Si pillars separated by $3 \mu \mathrm{m}$ trenches. First, the 8 - 
TABLE I. Summary of the structures analyzed by the different characterization techniques throughout this work. All the detailed substrates were coated with a $2-\mu$ m-thick Ge intermediate layer prior to the GaAs deposition. The indicated GaAs thicknesses correspond to the nominal values, but the real ones may vary depending on the pattern size.

\begin{tabular}{|c|c|c|c|}
\hline & Substrate & Pattern & GaAs thickness \\
\hline TDD of Ge virtual substrates & Offcut $\mathrm{Si}(001)$ & $2-15 \mu \mathrm{m}$ wide pillars & $\ldots$ \\
\hline \multirow[t]{4}{*}{ Morphological characterization by SEM } & Offcut $\mathrm{Si}(001)$ & $-10 \mu \mathrm{m}$ wide ridges & \\
\hline & & $-2-15 \mu \mathrm{m}$ wide pillars & $2 \mu \mathrm{m}$ \\
\hline & & $-15 \times 30 \mu \mathrm{m}^{2}$ rectangles & \\
\hline & & $-15 \mu \mathrm{m}$ wide pillars & $2-6 \mu \mathrm{m}$ \\
\hline Growth kinetics (AlAs markers) & Offcut Si (001) & $-15 \mu \mathrm{m}$ wide pillars & $4 \mu \mathrm{m}$, AlAs marker layers spaced $400 \mathrm{~nm}$ \\
\hline \multirow[t]{2}{*}{ TEM } & Offcut $\mathrm{Si}(001)$ & $-15 \mu \mathrm{m}$ wide pillars & $4 \mu \mathrm{m}$ \\
\hline & On-axis $\mathrm{Si}(001)$ & $-2 \mu \mathrm{m}$ wide pillars & $2 \mu \mathrm{m}$ \\
\hline \multirow[t]{3}{*}{ HRXRD } & Offcut $\mathrm{Si}(001)$ & $-10 \mu \mathrm{m}$ wide ridges & \\
\hline & & $-2-40 \mu \mathrm{m}$ wide pillars & $2 \mu \mathrm{m}$ \\
\hline & & $-15 \mu \mathrm{m}$ wide pillars & $2-6 \mu \mathrm{m}$ \\
\hline
\end{tabular}

$\mu$ m-high $\mathrm{Si}$ pillars are defined by photolithography and DRIE. The characteristic residual ripples from the Bosch process can be identified in the Si pillar sidewalls (Fig. 1(a)). Then, in order to adapt the lattice parameter and thermal strain mismatch between GaAs and $\mathrm{Si}, 2-\mu$ m-thick Ge virtual substrates are deposited by LEPECVD on top of the $\mathrm{Si}$

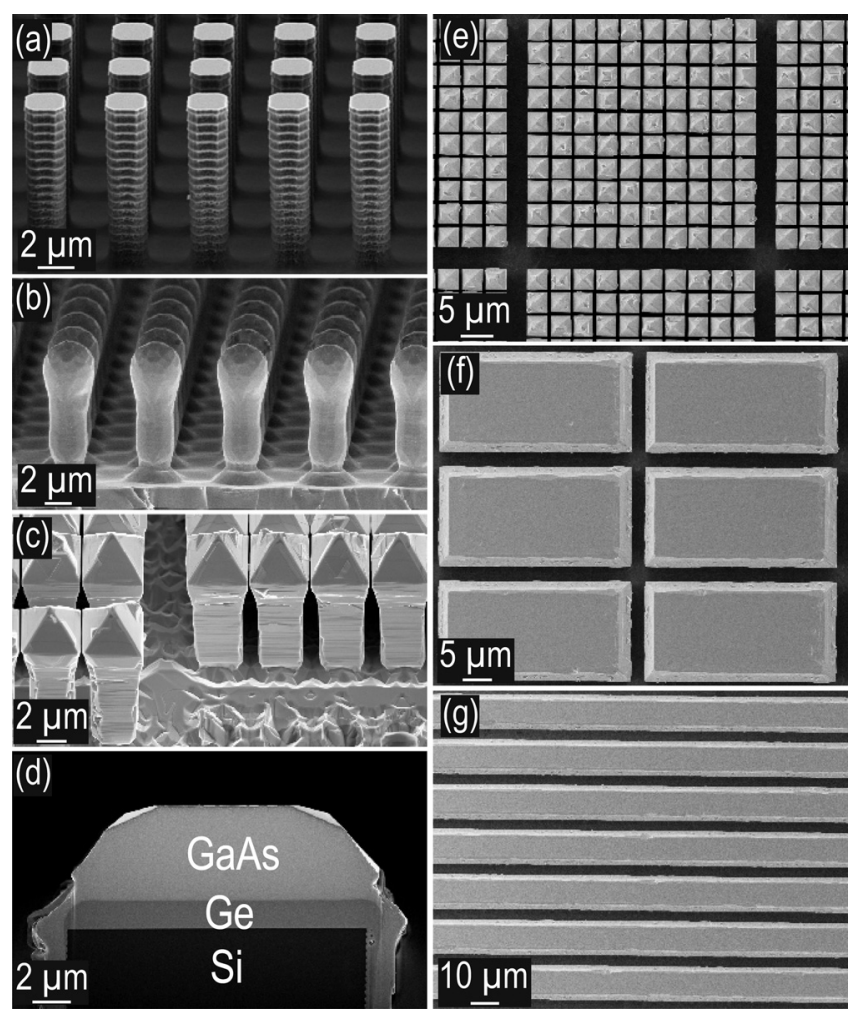

FIG. 1. (a)-(c) Perspective SEM micrographs of the main intermediate fabrication steps during the GaAs/Ge crystal growth on top of 2- $\mu$ m-wide Si pillars: (a) First, an array of $8-\mu \mathrm{m}$ in height Si pillars separated by $3 \mu \mathrm{m}$ trenches is micro-machined by DRIE. (b) After the growth of $2 \mu \mathrm{m}$ of Ge by LEPECVD and a post-growth annealing step, the Ge virtual $\mu$ m-substrates are defined. (c) Finally, $2 \mu \mathrm{m}$ of GaAs are deposited by MOVPE at $\mathrm{T}=680^{\circ} \mathrm{C}$. (d) SEM image of a FIB cross-section on the (110) plane of a $4-\mu \mathrm{m}$-tall GaAs crystal grown on top of a $15-\mu$ m-wide $\mathrm{Si}$ pillar previously coated with $2 \mu \mathrm{m}$ of Ge. The GaAs/Ge crystals can be integrated on top of $\mathrm{Si}$ patterns exhibiting different geometries, such as (e) 2- $\mu$ m-wide pillars separated by a $3 \mu \mathrm{m}$ gap and ordered in $10 \times 10$ blocks; (f) $30 \times 15 \mu \mathrm{m}^{2} \mathrm{Si}$ rectangles; or (g) 10- $\mu \mathrm{m}$-wide Si ridges along the [110]. pillars (Fig. 1(b)) and annealed. The Ge growth leads to the formation of a faceted crystal on the pillar top surface and a quasi-conformal growth on the Si rippled sidewalls. Finally, the GaAs is grown by MOVPE on top of the Ge/Si structures. The fabrication process results in an array of GaAs pyramidal crystals for this particular Si pillar geometry (Fig. 1(c)). A cross section parallel to (110) of a 4- $\mu$ m-tall GaAs crystal grown on top of a $15-\mu$ m-wide $\mathrm{Si}$ pillar is displayed in Figure 1(d). The image contrast permits to identify the Ge intermediate layer deposited on top of the Si pillar. A few examples of $\mathrm{GaAs} / \mathrm{Ge}$ crystals integrated on top of $\mathrm{Si}$ patterns exhibiting different geometries are presented in Figures 1(e)-1(g): 2- $\mu \mathrm{m}$-wide pillars separated by a $3 \mu \mathrm{m}$ gap and arranged in $10 \times 10$ blocks (Fig. $1(\mathrm{e})$ ), $30 \times 15 \mu \mathrm{m}^{2} \mathrm{Si}$ rectangles (Fig. 1(f)), and 10- $\mu \mathrm{m}$-wide Si ridges along [110] (Fig. 1(g)).

\section{A. Ge virtual $\mu$-substrates}

Figures 2(a)-2(d) show top SEM images of 2- $\mu$ m-tall Ge crystals grown on top of Si pillars ranging from 2 to $15 \mu \mathrm{m}$ in width. The Ge crystal facet distribution is highlighted in Fig. 2(b). The Ge crystal surface is mainly composed of (001), \{111\}, \{113\}, and $\left\{\begin{array}{lll}15 & 323\} \\ \text { facets. A }\end{array}\right.$ detailed description of the growth, morphological, structural, and optical properties of the Ge/Si crystals can be found elsewhere. ${ }^{30}$ As explained above, the miniaturization of the substrates down to the micron scale entails certain characteristic benefits like the ART and faceting for the TDD reduction. Even for $2-\mu \mathrm{m}$-tall Ge crystals, the TDD is reduced by increasing the ratio between the Ge crystal height and the $\mathrm{Si}$ base, as is displayed in Figure 2(e). Further increments of the Ge virtual substrate thickness lead to a more remarkable TDD reduction, even down to zero. This permits the growth of perfect Ge crystals from the highly defected interface with $\mathrm{Si}$, where a high density of misfit dislocations is still present. ${ }^{36}$ However, the Ge crystals evolve following their natural crystallographic directions and after a few microns the artificial offcut surface vanishes. ${ }^{19,31}$ In order to maintain this offcut surface, necessary for avoiding the APD formation during GaAs nucleation, the thickness of the Ge virtual $\mu \mathrm{m}$-substrates was limited to $2 \mu \mathrm{m}$. 


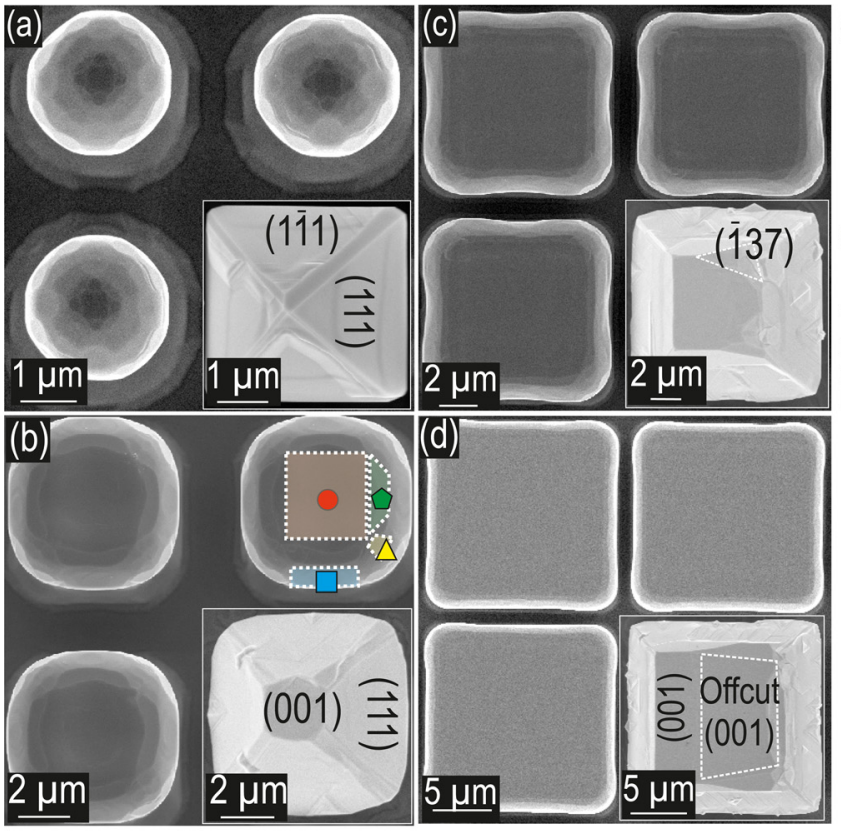

\section{B. GaAs crystals morphology and growth kinetics}

The insets of Figures 2(a)-2(d) show in more detail the morphology of the GaAs crystals corresponding to each Si pillar width after the MOVPE growth. The GaAs full pyramids grown on $2 \times 2 \mu \mathrm{m}^{2} \mathrm{Ge} / \mathrm{Si}$ crystals are terminated by $\{111\}$ facets. Nevertheless, GaAs/Ge crystals grown on larger Si pillars show a different facet distribution where surfaces belonging to the $\{137\}$ and $\{001\}$ family planes coexist with the main $\{111\}$ facets in the GaAs truncated pyramids (insets of Figs. 2(b)-2(d)). This GaAs facet distribution suggests an evolution towards a pyramidal shape due to the lower surface energy of $\{111\}$

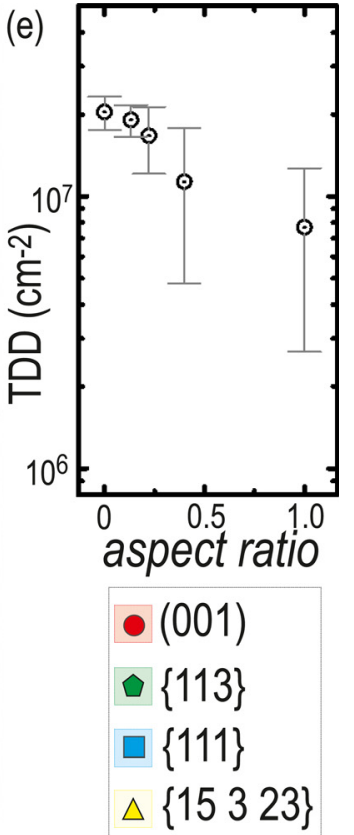

FIG. 2. (a) Top SEM view of $2-\mu$ m-tall Ge crystals grown on top of $8-\mu \mathrm{m}$-tall Si pillars etched into $\mathrm{Si}(001)$ substrates offcut $6^{\circ}$ towards [110]. The Si pillar sizes are $2 \times 2 \mu \mathrm{m}^{2}$ (a), $5 \times 5 \mu \mathrm{m}^{2}$ (b), $9 \times 9 \mu \mathrm{m}^{2}$ (c), and $15 \times 15 \mu \mathrm{m}^{2}$ (d). The facet distribution of the Ge crystals is indicated in (b). The insets show the morphology of the $2-\mu$ m-tall GaAs structures grown on top of the $\mathrm{Ge}$ virtual $\mu$ m-substrates. (e) TDD reduction by increasing the ratio between the Ge crystal height and the Si pillar lateral size.

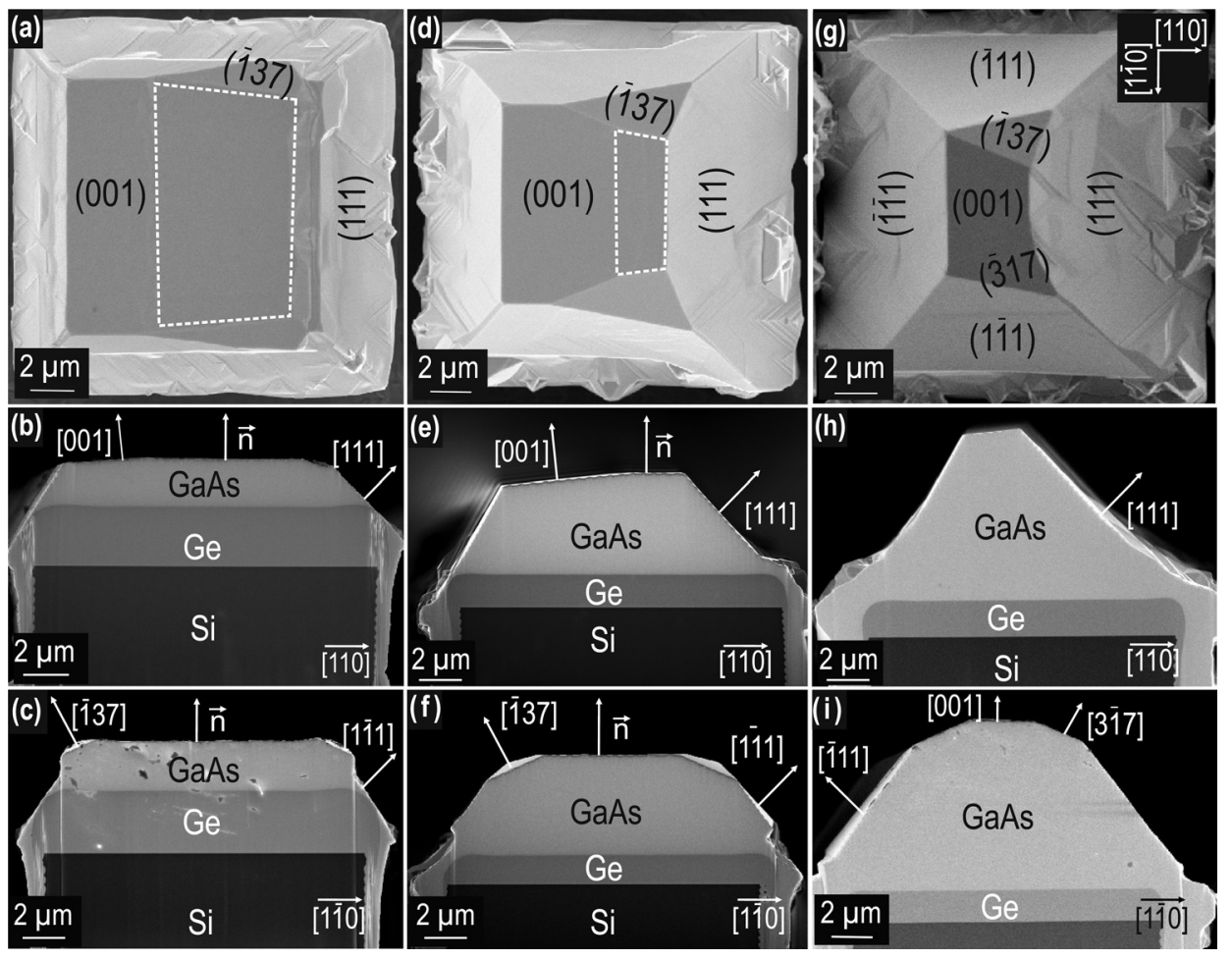

FIG. 3. Top surface and cross sections parallel to the (1-10) and (110) planes SEM images of (a)-(c) nominally $2 \mu \mathrm{m}$, (d)-(f) $4 \mu \mathrm{m}$, and (g)-(i) $6 \mu \mathrm{m}$ tall GaAs crystals grown on top of $15-\mu \mathrm{m}$-wide Si pillars. Prior to the GaAs growth, $2 \mu \mathrm{m}$ of Ge were deposited by LEPECVD. The FIB cross sections display the strong morphological asymmetry of $\mathrm{GaAs} / \mathrm{Ge}$ crystals grown on offcut Si patterned substrates. The direction normal to the Si offcut surface is indicated by the vector $\vec{n}$. 

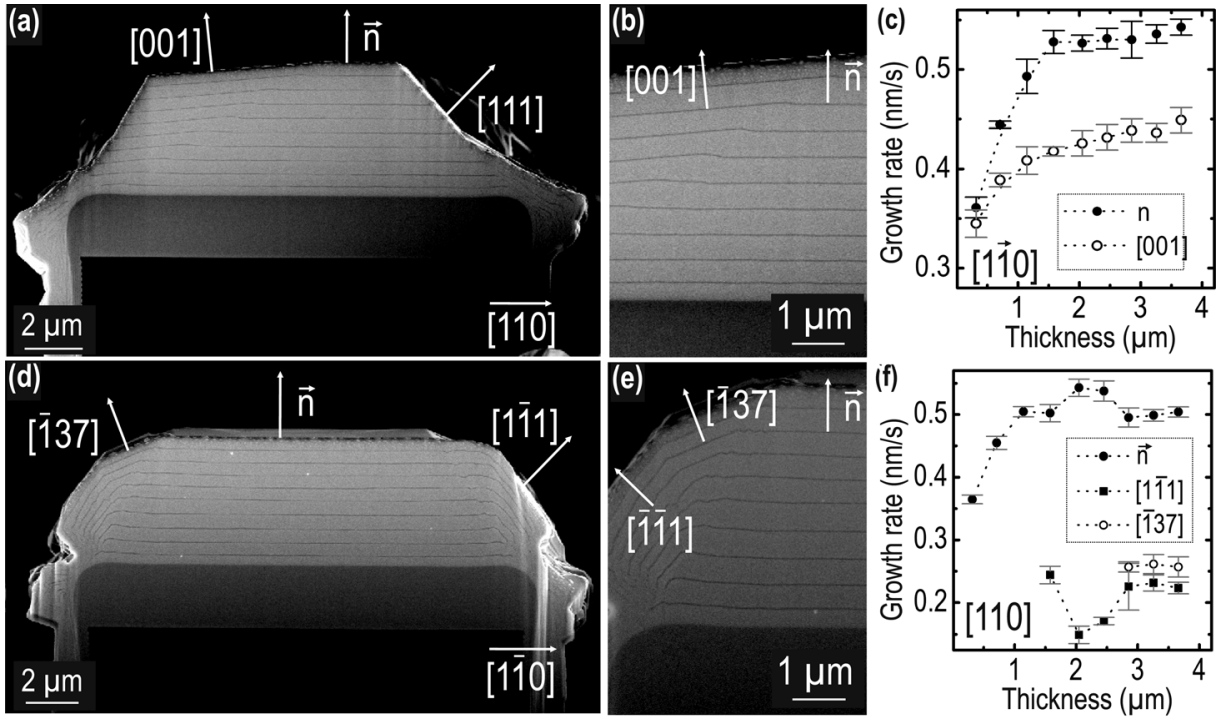

FIG. 4. 4- $\mu$ m-tall GaAs/Ge crystal grown on $15-\mu \mathrm{m}$-wide $\mathrm{Si}$ pillar. Its growth kinetics was traced by embedding 15-nm-thick AlAs marker layers spaced $400 \mathrm{~nm}$ during growth. (a) SEM cross section parallel to (1-10). The different evolution of the (001) facet and the surface parallel is shown in detail in (b). The growth rates perpendicular to both facets are shown in (c). Analogously, in (d) is shown a cross section of the $\mathrm{GaAs} / \mathrm{Ge} / \mathrm{Si}$ structure parallel to (110). The evolution of the $(-1-11)$ and (-137) facets is show in higher magnification in (e). (f) Growth rates in the directions perpendicular to the facets. Again, the vector $\vec{n}$ indicates the direction normal to the offcut surface.
(001) facet and a surface parallel to the offcut direction. The latter ones occupy $33 \%$ and $67 \%$ of the GaAs crystal top surface, respectively. Two incipient $\{137\}$ facets symmetrically arranged with respect to the [110] direction can be identified as well (Fig. 3(a)). The asymmetry of the GaAs crystals is evident in the FIB cross sections parallel to (1-10) and (110) displayed in Figures 3(b) and 3(c). An increment of the nominal thickness to $4 \mu \mathrm{m}$ leads to a clear evolution towards the pyramidal shape, due to the larger stability of the $\{111\}$ facets. Several changes of the morphology of the GaAs crystal apex can be identified in Fig. 3(d). The (001) facet prevails over the surface parallel to the offcut substrate, occupying $75 \%$ of the top GaAs crystal surface. The rotation of the growth axes towards the GaAs [001] natural direction is evident in the FIB cross sections parallel to the (1-10) planes, in contrast to the perpendicular one (110) (Figs. 3(e) and 3(f)). After the growth of a nominal thickness of $6 \mu \mathrm{m}$, the GaAs crystals have almost completed the evolution towards the final pyramidal shape. No remaining surface parallel to the offcut planes is present on the GaAs crystal top which, after $6 \mu \mathrm{m}$, is composed of a (001) facet and two $\{137\}$ facets arranged symmetrically with respect to the [110] GaAs crystal axes (Figs. 3(g) and 3(i)).

As a clear consequence of the unequal facet stability during growth, the thicknesses of the GaAs crystals along [001] differ to a large extent from the ones measured in planar reference samples, which are accurate to within $2 \%$ for nominally $6-\mu \mathrm{m}$-thick layers. The actual thickness values are $8.2 \%, 20 \%$, and $41.2 \%$ larger than the ones measured in planar substrates for GaAs crystals with 2, 4, and $6 \mu \mathrm{m}$ nominal thickness, respectively. This is a clear indication of Ga adatom migration from the stable $\{111\}$ facets to the top of the crystals during growth.

In order to analyze the GaAs growth kinetics on patterned substrates, 15-nm-thick AlAs marker layers spaced $400 \mathrm{~nm}$ were embedded into the GaAs crystals during MOVPE growth. The diffusion length of the group III adatoms decreases from $\mathrm{Ga}$ to $\mathrm{Al},{ }^{38}$ so that the introduction of the thin AlAs marker layers may slightly affect the growth kinetics of the GaAs crystals. Therefore, the facet growth rates and morphology of the crystals with embedded AlAs markers may differ to a small extent from the ones of pure GaAs crystals. Figures 4(a) and 4(b) show FIB cross sections parallel to the (1-10) and Figures 4(d) and 4(e) parallel to the (110) planes, respectively. Measuring the distance between AlAs markers in various growth directions allows the different facets growth rates to be estimated, which, as discussed below, is a key point in the morphological evolution of GaAs crystals.

The actual growth rates measured from FIB cross sections parallel to the (1-10) and (110) planes are displayed in Figures 4(c) and 4(f). The growth rate in the direction perpendicular to the offcut substrate is approximately $20 \%$ higher than that along the normal of the more stable (001) plane (Fig. 4(c)). This is in good agreement with the previously shown prevalence of the (001) facet with respect to the offcut surface (001). No growth can be identified both in the [111] and the [-1-11] crystal directions, due to the large stability of the GaAs $\{111\} \mathrm{B}$ facets. Here, we embrace the well-known convention that specifies GaAs (111)A as the orientation where the As and Ga surface atoms are bound, respectively, by one and three backbond(s) to the bulk GaAs. Conversely, the As atoms in the GaAs (111)B surface have three bonds with the GaAs bulk, while Ga atoms have one. Note that the GaAs (111)A and B facets of the $\mathrm{GaAs} / \mathrm{Ge} / \mathrm{Si}$ crystals are labeled with respect to the Miller indices of the Si(001) substrate. When compared with the conventional indexing for GaAs this therefore indicates a rotation of the GaAs crystal by $90^{\circ}$ with respect to the substrate. Such a crystal rotation has previously been related by several groups with the orientation of the $\mathrm{As}_{2}$ dimers during the nucleation process. ${ }^{15,39}$ The (1-11), (-111), (-137), (3-17) (001), and offcut (001) growth rates extracted from the FIB cross section parallel to the (110) planes are compared in Figure 4(f). The growth rates perpendicular to the (1-11) (GaAs $\{111\} \mathrm{A}$ in this case) and $(-137)$ facets are approximately a factor 2 lower than in the offcut [001] direction. 
It is, indeed, clear that the driving force for the GaAs pyramid formation is the large stability of the $\{111\}$ facets compared with the (001) facet and the surface parallel to the offcut plane. In particular, the $\{111\} \mathrm{B}$ facets are extremely stable, resulting in a negligible growth rate, the top (001) has the highest growth rate and $\{111\} \mathrm{A}$ and $\{137\}$ are characterized by intermediate growth rates. The coexistence of different facets in a GaAs crystal of dimensions on the micrometer scale amplifies these growth rate differences due to Ga adatom surface diffusion from the most stable facets towards the less stable ones during growth. The evolution of the GaAs crystals towards this low-energy facet distribution is an indication of the thermodynamic character of the MOVPE growth for the selected conditions, closer to equilibrium than, e.g., previously reported Ge crystal growth by LEPCVD on similarly patterned Si substrates. ${ }^{19}$

However, from the investigation of the growth kinetics several indications can be extracted regarding the strong morphological asymmetry of GaAs crystals grown on Ge coated Si pillars. First, the rotation of the crystals towards the GaAs natural crystallographic [001] direction may be understood by considering the large stability of the (001) facet compared with the offcut substrate surface plane. Second, the lack of equivalence of GaAs zinc-blende facecentered cubic sublattices becomes more evident in GaAs $\mu$ m-crystals. Within a scale of several micrometers, GaAs $\{111\} \mathrm{A}$ and GaAs $\{111\} \mathrm{B}$ facets coexist, and their characteristic different growth rates provide another source of morphological anisotropy. It seems plausible as well that the combination of both effects-the offcut related rotation and the difference in $\{111\} \mathrm{A}$ and $\{111\} \mathrm{B}$ GaAs facet kineticsleads to the formation of the $\{137\}$ facets symmetric to the [110] direction.

A composition of several bright-field TEM cross-section images acquired in the [2-20] Bragg condition, mapping a $4-\mu \mathrm{m}$-tall GaAs/Ge crystal grown on a $15-\mu \mathrm{m}$-wide Si pillar, is presented in Fig. 5(a). The large lattice mismatch between $\mathrm{Ge}$ and $\mathrm{Si}$ leads to the formation of a large density of misfit and threading dislocations near the interface of both materials. Due to the insufficient Ge thickness, the ART process is not completely efficient, and some of the threading dislocations reach the $\mathrm{GaAs} / \mathrm{Ge}$ interface, leading to the formation of new dislocations gliding into the GaAs. Furthermore, the GaAs crystal height is well above the critical thickness of the $\mathrm{GaAs} / \mathrm{Ge}$ system, so the strain at the interface is released by the formation of additional misfit dislocations. Interestingly, several clear examples of dislocation deflection by faceting can be seen in Fig. 5(a). This effect is even clearer in the TEM bright field image shown in Figure 5(b). In this case, we display a GaAs/Ge crystal grown on a $2-\mu$ m-wide pillar fabricated in an on-axis $\mathrm{Si}(001)$ substrate. Again, the interface between $\mathrm{Ge}$ and $\mathrm{Si}$ is heavily dislocated (Fig. 5(c)). Nevertheless, the higher aspect ratio of the Ge crystal leads to more efficient dislocation deflection through the sidewalls, such that only a small fraction of threading dislocations reaches the GaAs/Ge interface (Fig. 5(d)). Remarkably, no signatures of APD were found at the GaAs/Ge interface. This could be explained by the small size of the Ge crystal

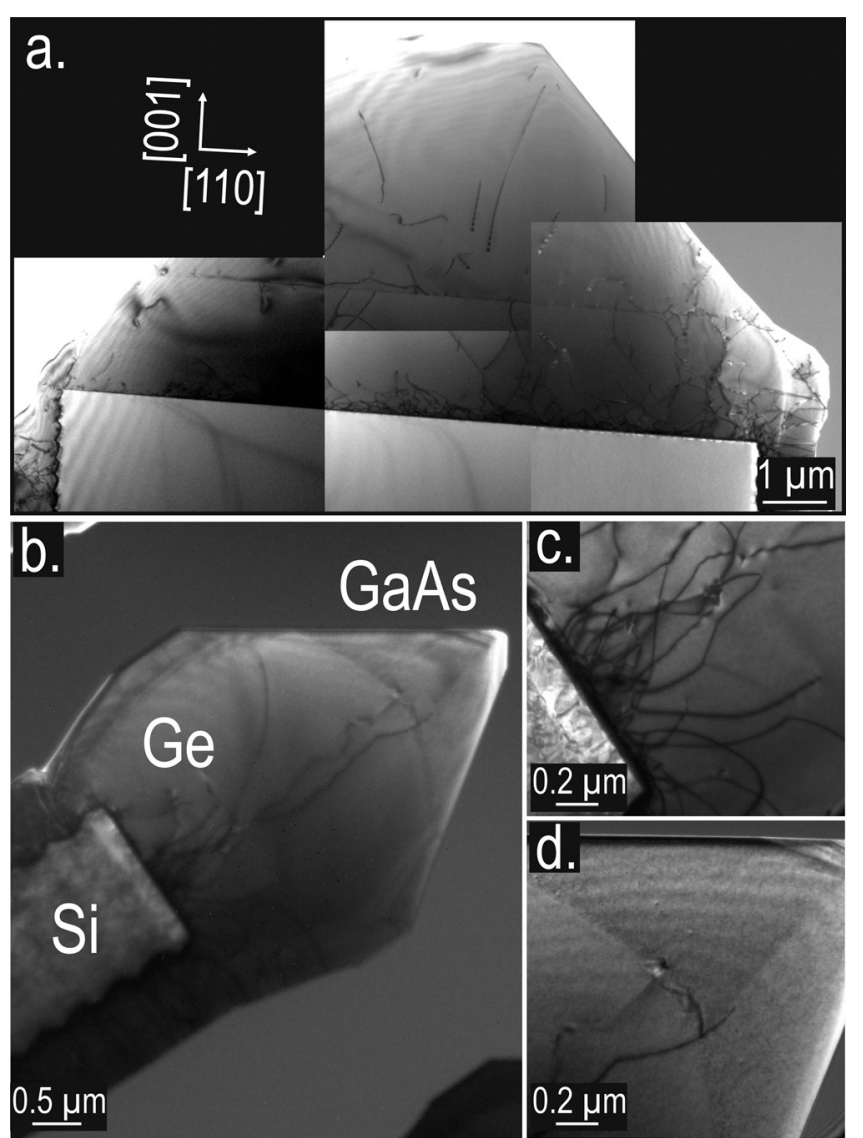

FIG. 5. (a) Composition of several bright-field TEM images mapping a 4- $\mu \mathrm{m}$-tall GaAs/Ge crystal grown on a $15-\mu \mathrm{m}$-wide Si pillar. (b) Bright-field TEM image of a GaAs/Ge crystal grown on a $2-\mu$ m-wide pillar fabricated in an on-axis $\mathrm{Si}(001)$ substrate. TEM micrographs recorded with higher magnification close to the $\mathrm{Ge} / \mathrm{Si}$ interface and the $\mathrm{GaAs} / \mathrm{Ge}$ interface are shown in (c) and (d), respectively.

top (001) facet that may facilitate the nucleation of a single GaAs domain.

\section{GaAs/Ge $\mu \mathrm{m}$-crystal structure: Substrate orientation and strain relaxation}

In the first stages of heteroepitaxial growth, the lattice mismatch between the grown materials and the substrate may be relaxed, among other processes, by the formation of an array of misfit dislocations. The range of both the Ge and GaAs crystal thicknesses studied in this paragraph is well above the critical thickness for misfit strain relaxation after their nucleation on $\mathrm{Si}$ and $\mathrm{Ge}$, respectively. Thus, the relaxation process studied here relates to the strain arising from the difference of the thermal expansion coefficients between both GaAs and Ge with $\mathrm{Si}$. This mismatch leads to an undesired tensile strain accumulation in the GaAs/Ge layers during the cooling down from the growth temperature to room temperature.

Moreover, the $\mathrm{Si}$ substrate misorientation has a strong effect on the crystallographic properties of the GaAs/Ge crystals, particularly on their tilt with respect to the substrate. Several authors reported on the tilt dependence on the initial substrate orientation, post-growth annealing, and epitaxial layer thickness. ${ }^{40}$ The accumulation of tilt during heteroepitaxial 
growth on misoriented substrates was initially explained by a coherent relaxation of the misfit strain due to the high density of steps along the offcut direction. ${ }^{41}$ In the first models, the tilt was understood as a geometrical consequence of misfit strain relaxation at atomic steps, but no effect of the plastic relaxation was considered. Subsequent models accounted for the presence of misfit dislocations, concluding that the tilt was generated by $60^{\circ}$ dislocations with Burgers' vector components orthogonal to the semiconductor/offcut-substrate interface. ${ }^{42}$ The origin of the tilt was attributed to the formation of $60^{\circ}$ dislocations during the coalescence of islands in the GaAs/Si system. ${ }^{43}$ Ayers et al. complemented the description of tilted epitaxy while they consider the preferential glide of certain Burgers' orientation due to the unequal stressing caused by the offcut. ${ }^{44}$

The previously described particularities of heteroepitaxial growth on offcut substrates strongly affect the structural properties of the material grown on Si patterned down to the micron scale. In this section, we present the structural characterization performed by HRXRD on GaAs/Ge crystals of different thicknesses grown on offcut $\mathrm{Si}$ substrates patterned with pillars of various sizes and geometries. The GaAs/Ge crystals were investigated by triple axis $\mathrm{X}$-ray diffraction measurements by collecting both (004) and (224) reciprocal space maps (RSM). The combined measurements of these two reflections can provide the perpendicular and parallel lattice constants, besides yielding valuable information on the lattice tilt, degree of relaxation, and mosaicity of the crystals. We will focus our attention on two main issues: first, the combined effect of growth on patterned substrates and the misorientation on the lattice deformation; finally, the relation of the thermal strain release mechanisms with the crystal aspect ratio (defined as the total GaAs/Ge height over the Si pillar width, in this case).

\section{Substrate orientation}

Epitaxial GaAs/Ge layers were grown on three different substrates for this set of experiments: $\mathrm{Si}$ wafers patterned with $9 \times 9-\mu \mathrm{m}^{2}$ pillars, $10-\mu \mathrm{m}$-wide ridges along the offcut direction and finally planar substrates. All the studied substrates had an offcut of $6^{\circ}$ towards [110]. The nominal thicknesses were $2 \mu \mathrm{m}$ of GaAs and $2 \mu \mathrm{m}$ of Ge in all three cases. The high epitaxial quality of $\mathrm{GaAs} / \mathrm{Ge} / \mathrm{Si}$ material grown by MOVPE was confirmed by the measurements, both for planar and patterned substrates. The HRXRD scans and RSM were measured in two different configurations, with the scattering plane along the [1-10], and with the scattering plane along the offcut direction, [110]. We define $\alpha$ as the angle between the X-ray scattering plane projection on the semiconductor surface and the offcut direction. Therefore, when the scattering plane projection is aligned with the [110] offcut direction, $\alpha=0^{\circ}$. Conversely, when it is aligned with the Si [1-10] direction, $\alpha=90^{\circ}$.

The RSM acquired around the symmetric (004) diffraction reciprocal lattice point (RLP) of GaAs/Ge layers grown on a planar Si substrate for azimuths $\alpha=90^{\circ}$ and $\alpha=0^{\circ}$ are shown in Figures 6(a) and 6(b), respectively. The two well separated diffraction peaks found for the planar material correspond to GaAs (A) and Ge (B). Two symmetric wings
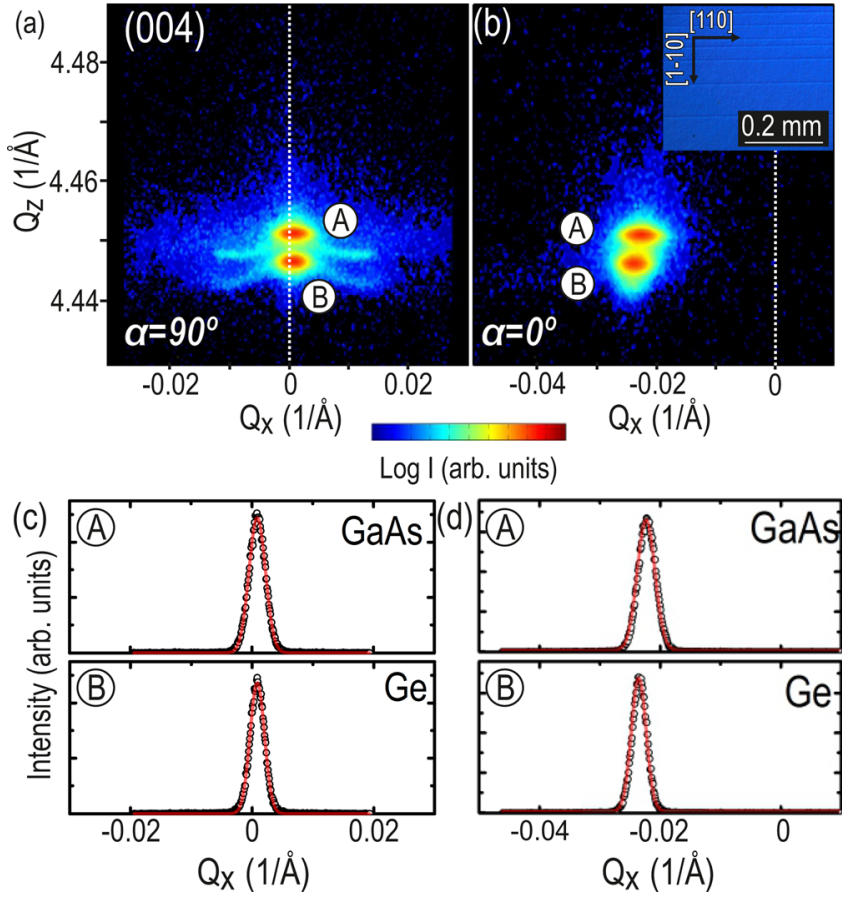

FIG. 6. RSM of a 2- $\mu$ m-thick GaAs film grown on top of a planar offcut $\mathrm{Si}$ substrate previously coated with $2 \mu \mathrm{m}$ of Ge. The presented RSMs were recorded around the (004) reciprocal lattice point (RLP) with the scattering plane defined by (a) the [001] and [1-10] directions $\left(\alpha=90^{\circ}\right)$ and (b) the [001] and the [110] offcut direction $\left(\alpha=0^{\circ}\right)$. The diffraction peaks corresponding to GaAs and $\mathrm{Ge}$ are labeled with the capital A and B letters, respectively. The Omega scans along to the GaAs and Ge diffraction peaks recorded with the scattering plane along [1-10] and [110] are presented in (c) and (d), respectively.

along $Q_{x}$ are visible around both GaAs and Ge when $\alpha=90^{\circ}$. They result from weak diffuse scattering by the partially relaxed material near thermal cracks. ${ }^{45}$ In contrast, the symmetric wings are not present in the RSM measured with $\alpha=0^{\circ}$. This is in good agreement with the preferential formation of thermal cracks along the offcut direction that can be derived from Nomarski images of the GaAs surface (Fig. 6(b), inset). ${ }^{46}$ Scans along the $Q_{x}$ axis through the GaAs and Ge diffraction maxima are shown in Figures 6(c)-6(d) for $\alpha=90^{\circ}$ and $\alpha=0^{\circ}$, respectively. The Gaussian fits indicate small differences in the GaAs peak width along [110] and [1-10] $\left(\mathrm{FWHM}_{\left(\alpha=0^{\circ}\right)}=0.0033 \AA^{-1}\right.$ and $\left.\operatorname{FWHM}_{\left(\alpha=90^{\circ}\right)}=0.0031 \AA^{-1}\right)$. The tilt angles $\chi$ measured with respect to the Si substrate in both configurations are different as expected: $\chi_{\mathrm{GaAs}}=-0.281^{\circ}$ and $\mathrm{Ge} \chi_{\mathrm{Ge}}=-0.292^{\circ}$ with $\alpha=0^{\circ}$ and $\chi_{\mathrm{GaAs}}=0.012^{\circ}$ and $\mathrm{Ge} \chi_{\mathrm{Ge}}=0.012^{\circ}$ when the X-ray scattering plane is aligned with [1-10] $\left(\alpha=90^{\circ}\right)$.

The HRXRD characterization of the planar layers was completed with RSM recorded around the asymmetric (224) azimuth in both $\alpha=90^{\circ}$ and $\alpha=0^{\circ}$ configurations (not shown). The corresponding experimental values of the average parallel strain $\left(\epsilon_{\|}^{V}\right)$ for planar $\mathrm{GaAs}$ and $\mathrm{Ge}$ are $\epsilon_{\|, \alpha=90^{\circ}}^{V}(G a A s)=0.19 \%$ and $\epsilon_{\|, \alpha=90^{\circ}}^{V}(G e)=0.14 \%$ with $\alpha=90^{\circ}$. The calculated values with the scattering plane parallel to the offcut $\left(\alpha=0^{\circ}\right)$ are $\epsilon_{\|, \alpha=0^{\circ}}^{V}(G a A s)=0.16 \%$ and $\epsilon_{\|, \alpha=0^{\circ}}^{V}(G e)=0.15 \%$.

Figures 7(a) and 7(b) illustrate the RSM obtained from $\mathrm{GaAs} / \mathrm{Ge}$ crystals grown on top of $10-\mu \mathrm{m}$-wide Si ridges. Two 

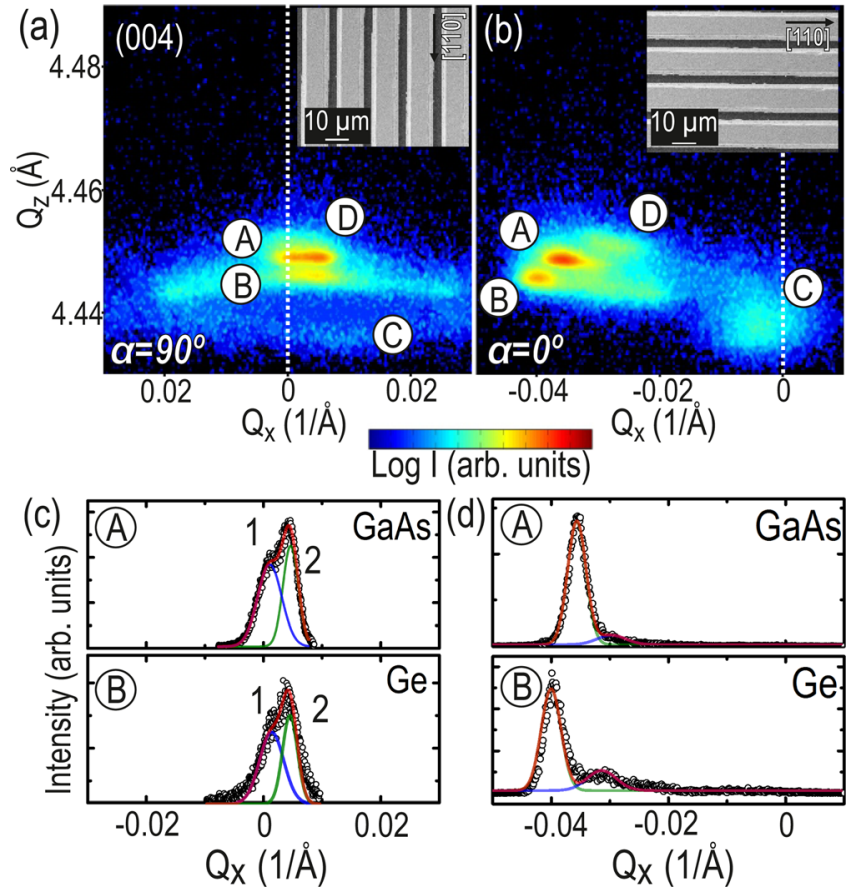

FIG. 7. RSM of a $2-\mu$ m-thick GaAs film grown on top of $10-\mu$ m-wide $\mathrm{Si}$ ridges. Before the GaAs growth, 2- $\mu \mathrm{m}$ of Ge was deposited in the patterned substrate by LEPECVD. The long dimension of the ridges is aligned with the offcut direction (insets). The RSMs were recorded around the (004) RLP with the scattering plane in the previously defined azimuth alignments $\alpha=90^{\circ}$ (a) and $\alpha=0^{\circ}$ (b). The omega scans corresponding to the GaAs and Ge diffraction peaks recorded at $\alpha=90^{\circ}$ and $\alpha=0^{\circ}$ are presented in (c) and (d), respectively.

clear reflections stemming from GaAs (A) and Ge (B) can be identified in the RSM around the (004) diffraction in both the azimuth $\alpha=90^{\circ}$ and $\alpha=0^{\circ}$ configurations. When $\alpha=0^{\circ}$ the $\mathrm{X}$-ray scattering plane faces the ridges along their long axis, so the contribution of the material in the vicinity of the top offcut surface predominates. In the $\alpha=0^{\circ}$ configuration, the lattice tilts calculated from the GaAs and Ge diffraction peaks are $\chi_{\mathrm{GaAs}}=-0.458^{\circ}$ and $\chi_{\mathrm{Ge}}=-0.511^{\circ}$, respectively, substantially larger than the ones measured in the planar substrates. When $\alpha=90^{\circ}$, the X-ray scattering contributions stemming from the lateral facets and the top offcut surface of the ridges add together to the diffraction pattern. A double peak structure was found while recording an $\omega$ scan around the GaAs (004) RLP (Fig. 7(c)). The corresponding tilt values were calculated by Gaussian fitting: $\chi(1)_{\mathrm{GaAs}}=0.021^{\circ}$ and $\chi(2)_{\mathrm{GaAs}}=0.059^{\circ}$ for $\mathrm{GaAs}$ and $\chi(1)_{\mathrm{Ge}}=0.019^{\circ}$ and $\chi(2)_{\mathrm{Ge}}=0.038^{\circ}$ for Ge. The analysis of the RSM around the asymmetric GaAs (224) reflection yields strain values: $\epsilon_{\|, \alpha=90^{\circ}}^{V}(G a A s)=0.06 \%, \epsilon_{\|, \alpha=90^{\circ}}^{V}$ $(G e)=0.10 \%$ in the $\alpha=90^{\circ}$ configuration and $\epsilon_{\|, \alpha=0^{\circ}}^{V}(G a A s)$ $=0.17 \%$ and $\epsilon_{\|, \alpha=0^{\circ}}(G e)=0.14 \%$ in the $\alpha=0^{\circ}$ configuration, in both cases the strain values correspond to the most intense contribution.

In addition, two low intensity contributions labelled by capital letters $\mathrm{C}$ and D can be found in both RSM of Figs. 7(a) and 7(b). We relate the (C)-contribution with residual material accumulated on the ridge sidewalls and the (D)-contribution with strained material deposited in the trenches between adjacent ridges. As pointed out earlier in this section, the differential relaxation of the epitaxial material during growth along the offcut direction leads to the accumulation of tilt with respect to the substrate. This applies also to the strained material in the $\mathrm{Si}$ (001) trenches (D), inducing the shift along $Q_{x}$ when rotating the X-ray scattering plane from $\alpha=90^{\circ}$ to $\alpha=0^{\circ}$. On the other hand, the diffraction maximum $\mathrm{C}$ is not affected by such a rotation. This indicates that it stems only from GaAs on the parts of the patterned $\mathrm{Si}$ substrate which is not affected by the offcut, in this case the $\mathrm{Si}$ (110) planes which form the sidewalls of ridges. These two low intensity diffraction peaks $\mathrm{C}$ and $\mathrm{D}$ are found in all the patterned structures studied in this work.

The complex structure of the GaAs/Ge crystals grown on $9 \times 9 \mu \mathrm{m}^{2}$ Si pillars is revealed in the RSM recorded in the vicinity of the $\mathrm{GaAs}(004)$ symmetric reflection for $\alpha=90^{\circ}$ (Fig. 8(a)). The small lattice mismatch between both materials, when they are relaxed, complicates the diffraction peak assignment. The different structure factor of the Ge and GaAs lattices allowed us to discriminate the diffraction collected from the two different materials by recording RSM around the (002) reflection (not shown). The two intense diffraction peaks labeled with the capital letters $A$ and $B$ in Figure 8(a) correspond to GaAs and Ge, respectively. Truncation rod scattering contributions stemming from the GaAs(111) facets can be found symmetrically distributed forming an angle $\beta \approx \pm 54^{\circ}$ with the $Q_{x}=0$ axis, starting their divergence at the GaAs(004) RLP. Diffuse diffraction signals similar to the ones found in the ridges case are labeled in the RSM with a capital $\mathrm{C}$.

Single scans along the $Q_{x}$ direction centered in the GaAs and Ge peaks are shown in Fig. 8(c) for $\alpha=90^{\circ}$. The Ge

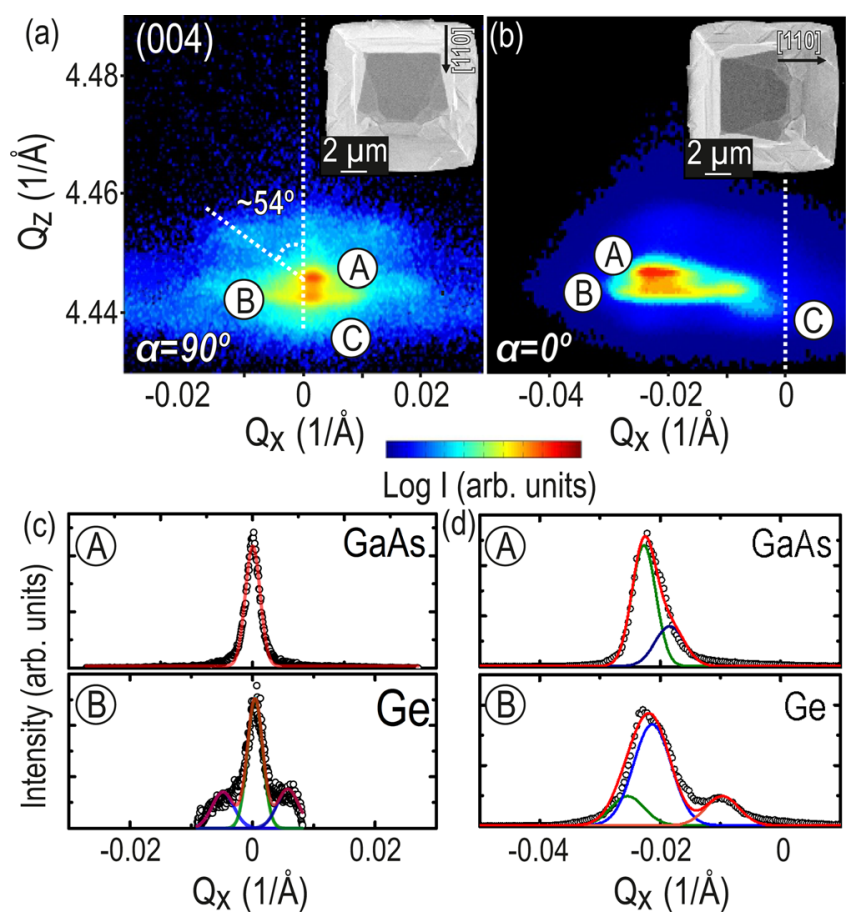

FIG. 8. RSM of GaAs/Ge crystals grown on arrays of 9- $\mu$ m-wide Si pillars with $\alpha=90^{\circ}$ (a) and $\alpha=0^{\circ}$ (b) The insets sketch the relative alignment of the X-ray scattering planes and the crystals. Omega scans along the $Q_{x}$ momentum of the GaAs and Ge diffraction peaks with $\alpha=90^{\circ}$ and $\alpha=0^{\circ}$ are shown in (c) and (d), respectively. 
diffraction presents a widening along the $Q_{x}$ momentum that can be fitted with three Gaussians. Each one may be related to a different lattice tilt with respect to the Si substrate. The main contribution shows a small tilt value of $\chi_{\mathrm{Ge}}=0.014^{\circ}$. The two satellite peaks, symmetrically arranged with respect to the most intense Ge diffraction yield tilt values of $\chi_{, 1}$ $=-0.049^{\circ}$ and $\chi_{, 2}=0.054^{\circ}$. The measured varying lattice tilts around the $\mathrm{Ge}(004)$ reflection are related to elastic accommodation of the thermal strain of material close to the interface through lattice bending across the diameter of the crystals. ${ }^{32}$ The FWHM of the Gaussian fitting the GaAs measured peak is as low as $0.0024 \AA^{-1}, \sim 30 \%$ smaller than the one obtained for the planar layers. A small lattice tilt with respect to $\mathrm{Si}(001)$ was measured in the $\mathrm{GaAs}$ $\left(\chi_{\mathrm{GaAs}}=0.015^{\circ}\right)$ when $\alpha=90^{\circ}$. It is worth mentioning that during the $\omega$ scans the X-ray diffraction is mainly sensitive to the distortion of the (001) crystal planes. It is known that dislocations can widen the $\omega$ scans as they introduce a rotation of the crystal and are surrounded by a strain field in which the Bragg angle of the crystal is non-uniform. ${ }^{47}$ The scattered X-ray intensity distribution of dislocated epitaxial material can be calculated within the framework of the kinematical diffraction theory. Several groups have faced this problem in planar films and related the HRXRD peaks width with the dislocation density and spatial correlation by means of Monte Carlo calculations. ${ }^{48-50}$ Nevertheless, the convolution of different effects in the GaAs/Ge crystals such as lattice bending at the interface, slight changes in the thermal strain release in the vicinity of the facets and rotation of the tilt axis may screen the dislocation related widening of the $\omega$ scan. Therefore, these advances cannot be directly applied to heteroepitaxial material grown on patterned substrates. The narrowing of the FWHM of $\omega$ scans measured in the GaAs/ Ge crystals should be then interpreted as a qualitative improvement of the crystal quality compared with planar material. An absolute defect density measurement would require further theoretical and experimental research, which is out of the scope of this work.

In order to quantify the strain of the GaAs/Ge crystals, the RSM around the GaAs(224) asymmetric reflection was measured. Again, the discrimination between the GaAs and Ge contributions to the diffraction pattern was carried out by recording the RSM around an azimuth selective to the GaAs and Ge structure factors, in this case the GaAs (226). Both the GaAs and Ge grown on the $9 \times 9 \mu \mathrm{m}^{2}$ Si pillars are relaxed, presenting in plane strain values as low as $\epsilon_{\|, \alpha=90^{\circ}}^{V}(G a A s)=0.013 \%$ and $\epsilon_{\|, \alpha=90^{\circ}}^{V}(G e)=0.022 \%$, respectively.

The RSM recorded around the GaAs(004) RLP after an orthogonal rotation of the $\mathrm{GaAs} / \mathrm{Ge} / \mathrm{Si}$ structures with respect to the X-ray scattering plane $\left(\alpha=0^{\circ}\right)$ are displayed in Fig. 8(b). Both the $\mathrm{GaAs}(004)$ and $\mathrm{Ge}(004)$ diffraction peaks are shifted towards lower, negative $Q_{x}$ momenta compared with the ones measured for $\alpha=90^{\circ}$ around the same reflections (Fig. 8(d)). The $\omega$ scan around the Ge RLP can be fitted by 3 Gaussian curves, in analogy to the $\alpha=90^{\circ}$ case. In contrast, the $\omega$ scan around the GaAs peak with $\alpha=0^{\circ}$ is asymmetric and wider than the one recorded in the perpendicular alignment. The variation of thickness along the crystal diameter shown in Figure 3 may lead to slight variations of the tilt leading to the observed widening of the $\omega$ peaks for $\alpha=0^{\circ}$ compared with the ones measured in the $\alpha=90^{\circ}$ configuration. Several groups have reported increments of the tilt with increasing thickness of GaAs and InGaAs layers grown on planar Si. ${ }^{51,52}$ As detailed in Section III B, the thickness of the "layers" grown on patterned substrates changes largely with the crystal morphology. Therefore, differences in the lattice tilt are expected between the various facets. The calculated tilts for the GaAs and $\mathrm{Ge}$ main components $\left(\chi_{\mathrm{GaAs}}=-0.276^{\circ}\right.$ and $\left.\mathrm{Ge} \chi_{\mathrm{Ge}}=-0.279^{\circ}\right)$ are similar to the ones measured in the planar material but remarkably smaller than the ones obtained from the analysis of the $\mathrm{GaAs} / \mathrm{Ge}$ ridges. The strain values obtained with $\alpha=0^{\circ}$ $\left(\epsilon_{\|, \alpha=0^{\circ}}^{V}(G a A s)=0.014 \%\right.$ and $\left.\epsilon_{\|, \alpha=0^{\circ}}^{V}(G e)=0.026 \%\right)$ are quite similar to the ones measured for $\alpha=90^{\circ}$.

The preceding observations based on the X-ray data portray valuable differences between the crystals grown on the three different substrates (planar, ridges, and pillars). Two of the analyzed structures, the planar layers and the GaAs/Ge crystals grown on $9 \times 9 \mu \mathrm{m}^{2}$ Si pillars undergo biaxial strain. The former accumulates a similar amount of strain in both [110] and [1-10] during cooling down from the growth temperature, while elastic relaxation of the thermal strain is found in the latter, as will be addressed in detail in Sec. III C2. On the other hand, the ridges present substantial differences in the strain relaxation process along their perpendicular dimensions. The thermal strain relaxes elastically along the direction perpendicular to the ridge main axis, while it does not in the parallel direction.

The GaAs/Ge ridge crystal lattice has a tilt distortion that is a factor 1.64 higher than the one measured both in the $\mathrm{GaAs} / \mathrm{Ge}$ crystals and planar layers when $\alpha=0^{\circ}$. Moreover, similar tilt values are found for both $\mathrm{Ge}$ and $\mathrm{GaAs}$ in the different structures studied apart from the ridges. As mentioned above, tilt originates from three sources in heteroepitaxial systems: the elastic contribution at the surface steps, the uneven distribution of $60^{\circ}$ misfit dislocations between the four possible $\{111\}$ slip planes, and the lattice bending to relax elastically the thermal strain. ${ }^{32}$ The slip plane inequality arises from the broken symmetry of the offcut substrate. The substrates are patterned well above the size that could influence the nucleation process of the GaAs/Ge crystals. This means that all the analyzed layers should accumulate a similar amount of tilt with respect to the substrate during the nucleation stage. In contrast, due to the distinct geometries of the analyzed patterns they accumulate different amounts of thermal strain. As stated above, in the two symmetric structures (the $9 \times 9 \mu \mathrm{m}^{2}$ pillars and the planar substrate) the thermal strain can be approximated to be biaxial. Conversely, in the $10-\mu \mathrm{m}$ wide ridges the thermal stress along the ridges is substantially larger than in their short dimension. This stress may be relaxed by a coherent deformation of the lattice or plastically, through the formation of dislocations. Both paths lead to a tilt accumulation with respect to the Si substrate. This effect must be larger in the $\mathrm{Ge}$, which is subjected to cyclic thermal annealing before the GaAs growth. It has been shown in the GaAs/Si system that residual dislocations are created during the cooling after growth, due to the thermal expansion mismatch, so the generation of tilt as a by-product of plastic strain relaxation of 
an anisotropic thermal stress is more plausible in this particular case. ${ }^{53}$

\section{Strain relaxation: Aspect ratio dependence}

Two different sets of experiments were completed in order to evaluate the strain relaxation process of $\mathrm{GaAs} / \mathrm{Ge}$ crystals with different aspect ratio. First, we evaluate the strain relaxation of GaAs/Ge crystals with a constant nominal height of $4 \mu \mathrm{m}$ ( $2 \mu \mathrm{m}$ of GaAs and $2 \mu \mathrm{m}$ of $\mathrm{Ge})$ grown on arrays of $\mathrm{Si}$ pillars varying in width between 2 and $40 \mu \mathrm{m}$. Then, the strain of the crystals grown on $15-\mu \mathrm{m}$-wide $\mathrm{Si}$ pillars is evaluated for a nominal GaAs thickness varying from 2 to $6 \mu \mathrm{m}$.

As discussed in Sec. III C 1, the parallel strain measured in crystals grown on $9 \times 9 \mu \mathrm{m}^{2}$ Si pillars was not affected by the substrate offcut. We have therefore limited the study of the strain relaxation process to one single alignment between the GaAs/Ge crystals and the X-ray scattering plane. The following discussion of the strain relaxation of $\mathrm{GaAs} / \mathrm{Ge} / \mathrm{Si}$ structures will be based on HRXRD scans and RSM recorded with the scattering plane along the [1-10] $\left(\alpha=90^{\circ}\right)$ around the GaAs (004) and (224) diffractions.

The RSM measured around the (004) diffraction RLP of GaAs/Ge crystals grown on patterned substrates formed by 40-, 15-, and 5- $\mu \mathrm{m}$-wide Si pillars are displayed in Figures 9(a)-9(c). Two intense peaks stemming from the GaAs and Ge were recorded for the different substrates. Both Ge and GaAs diffraction peaks shift to lower $Q_{z}$ momentum values when the aspect ratio of the structure increases, indicating a release of the thermal strain. Figure 9(d) compares the

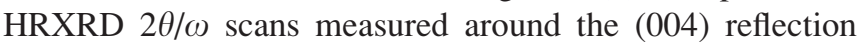
for GaAs/Ge crystals grown on Si pillars with widths ranging from 2 to $40 \mu \mathrm{m}$. The diffraction collected from a $(2+2)-\mu \mathrm{m}$-thick $\mathrm{GaAs} / \mathrm{Ge}$ layer grown on a planar $\mathrm{Si}(001)$ substrate is used as a reference. The curves in Figure 9(d) show a monotonic trend towards lower Bragg angles which confirms the elastic relaxation of the thermal strain in GaAs/ $\mathrm{Ge} / \mathrm{Si}$ heterostructures of high aspect ratio. The strain values were quantified following the same procedure detailed in Sec. III C1. A monotonically rising strain was found for increasing structure size up to the values observed for planar GaAs and Ge (Fig. 9(e)). The crystals grown on $5 \times 5 \mu \mathrm{m}^{2} \mathrm{Si}$ pillars exhibit strain values as low as $0.003 \%$ for GaAs and $0.001 \%$ for $\mathrm{Ge}$, revealing complete thermal strain relaxation.

In order to further interpret the experimental results, the strain obtained from HRXRD was compared with theoretical calculations. By means of the elasticity-theory FEM simulations introduced in Section II, we evaluated how the thermal strain components of the film-like structure were modified by the presence of the free pillar surfaces. In particular, an initial, constant elastic field was assumed for every material according to the measurements on the unpatterned region. Then, the strain field variation after considering the free-surface boundary condition was evaluated by simulations. The measured GaAs in-plane thermal strain in the unpatterned region $\left(\epsilon_{\|}^{V}(G a A s)=0.19 \%\right)$ was imposed as initial thermal strain in both the in-plane and the [001] directions. The same applied for $\mathrm{Ge},\left(\epsilon_{\|}^{V}(G e)=0.14 \%\right)$, where the experimental in-plane thermal strain is lower than the theoretical prediction due to a
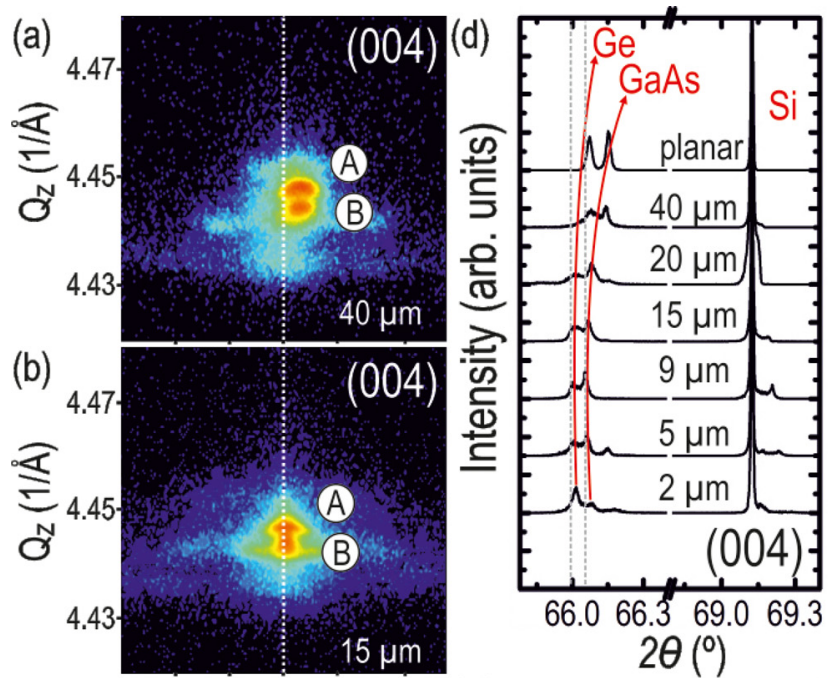

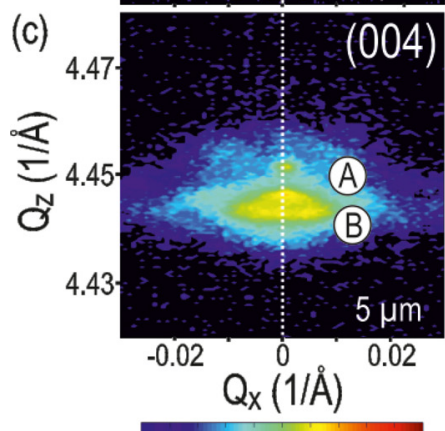

Log I (arb. units)

(f)

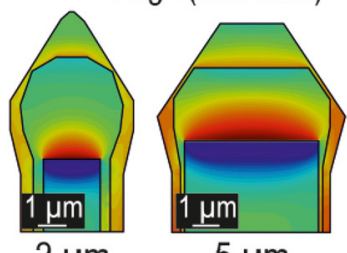

(g)

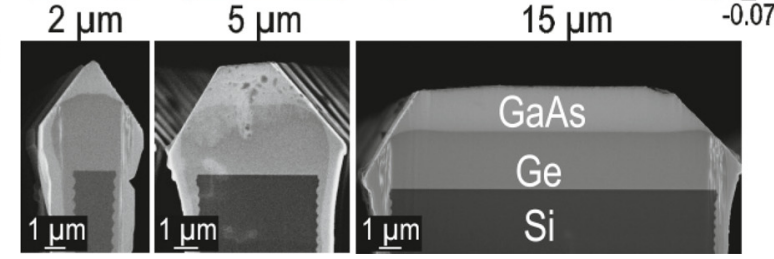

FIG. 9. RSM measured around the (004) RLP of 2- $\mu$ m-thick GaAs epilayers grown on arrays of 40- $\mu \mathrm{m}$ (a), $15-\mu \mathrm{m}$ (b), and 5- $\mu \mathrm{m}$-wide (c) Ge/Si pillars,

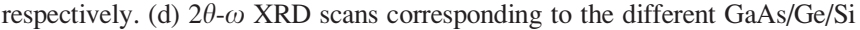
microstructure sizes (from 2 to $40 \mu \mathrm{m}$ in width) compared with reference epitaxy grown on a planar $\mathrm{Ge} / \mathrm{Si}$ substrate. The dashed lines indicate the relaxed GaAs and Ge diffraction angles. The red lines are guides to the eye. (e) Strain values for the different $\mathrm{GaAs} / \mathrm{Ge} / \mathrm{Si}$ microstructure sizes. (f) Strain relaxation maps obtained from FEM simulations for GaAs/Ge crystals on 2- and 15- $\mu$ mwide Si pillars. The strain maps in the simulated geometries are compared with their correspondent SEM cross-section images, displayed in (g).

low compressive residual misfit strain. ${ }^{54}$ Maps of the in plane thermal strain of three geometries related to $\mathrm{GaAs} / \mathrm{Ge}$ crystals grown on top of Si pillar bases of 2-, 5-, and 15- $\mu \mathrm{m}$ are shown in Figure 9(f). The corresponding SEM cross sections of the GaAs/Ge crystals (110) plane are displayed in Figure 9(g). The volume average of the in-plane strain from FEM simulations yielded strains of $0.011 \%, 0.015 \%$, and $0.031 \%$ for GaAs on 2, 5, and $15 \mu \mathrm{m}$ wide Si pillars. These values are in good agreement with the experimental strains from HRXRD (Fig. 9(e)). 
Finally, the strain relaxation process of GaAs/Ge crystals of different heights was investigated. RSMs recorded around the (004) reflection of nominal GaAs thicknesses of 6,4 , and $2 \mu \mathrm{m}$ grown on top of 15 - $\mu \mathrm{m}$-wide Si pillars arrays are shown in Figures 10(a)-10(c). A thickness increase induces a shift towards lower $Q_{z}$ momentum values, indicating again the release of crystal strain. Furthermore, the shift along the $Q_{z}$ direction makes both $\mathrm{Ge}$ and GaAs diffraction

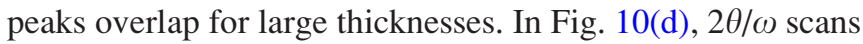
along the GaAs and $\mathrm{Ge}(004)$ diffraction peaks are presented and decomposed into three Gaussian curves corresponding to the contributions of GaAs, Ge, and the strained material accumulated in the trenches. Increments of the thickness lead to an enhancement of the GaAs peak intensity compared

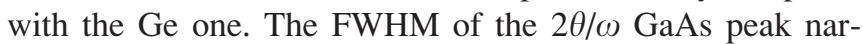
rows down from $120 \operatorname{arcsec}(2 \mu \mathrm{m}$ of GaAs) to $93.2 \mathrm{arc} \mathrm{sec}$

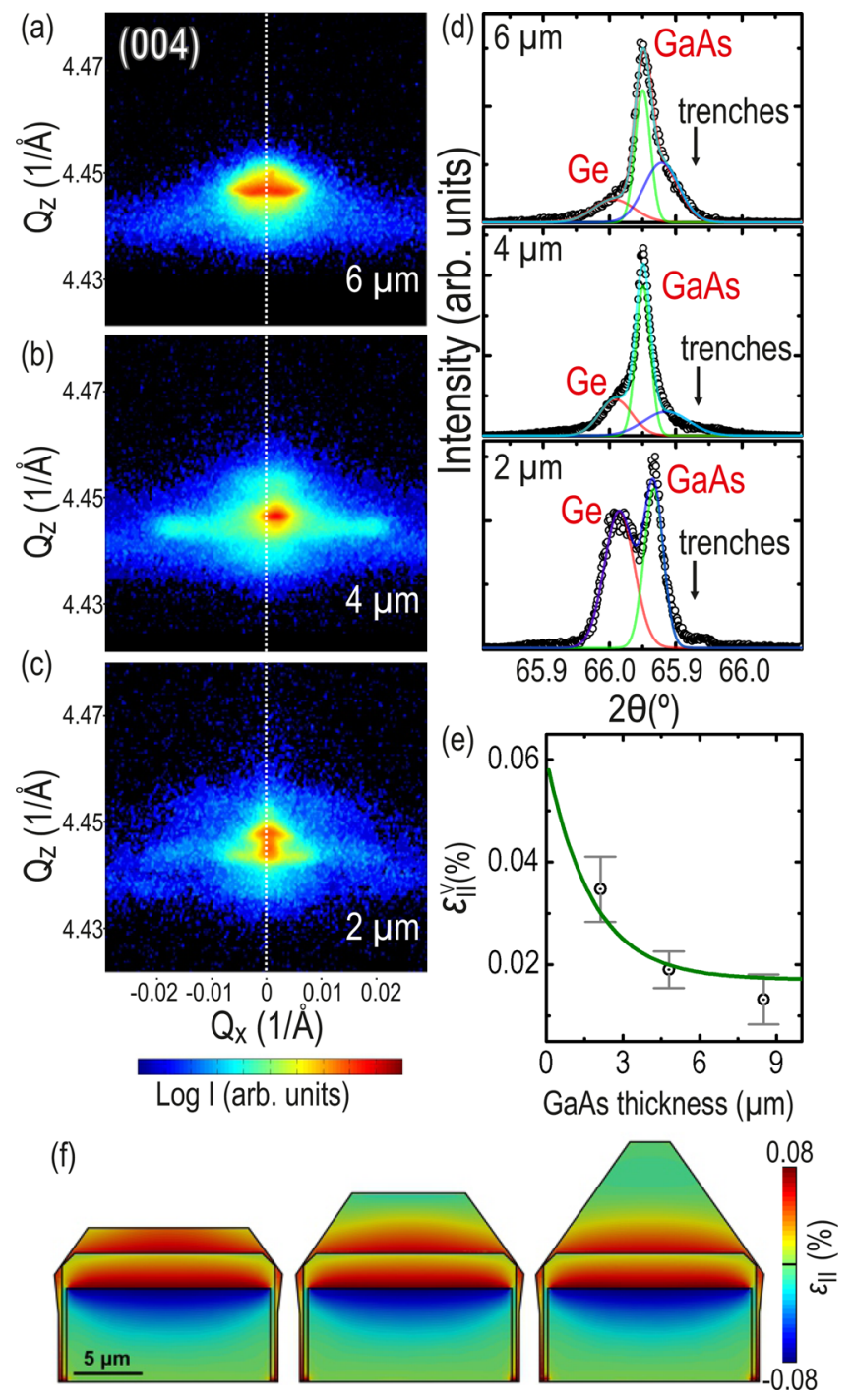

FIG. 10. RSM measured around the (004) RLP of (a) 6-, (b) 4-, and (c) 2$\mu$ m-thick GaAs epilayers grown on arrays of $15-\mu \mathrm{m}$ wide $\mathrm{Ge} / \mathrm{Si}$ pillars. (d) 2 $\theta-\omega$ XRD scans corresponding to the different GaAs thicknesses (from 2 to $6 \mu \mathrm{m}$ ). (e) Strain values for the different GaAs thickness. The solid line corresponds to the calculated exponential decay of the strain with the crystal thickness. (f) Strain relaxation maps obtained from FEM simulations for thick GaAs crystals grown on $15-\mu \mathrm{m}$-wide $\mathrm{Ge} / \mathrm{Si}$ pillars with nominal heights 6,4 , and $2 \mu \mathrm{m}$.
( $6 \mu \mathrm{m}$ of GaAs) indicating an improvement of the crystal quality when the thickness is increased. The increments in GaAs thickness lead to a widening along the $Q_{x}$ direction, as well. This widening is particularly evident in the $6-\mu \mathrm{m}$-tall GaAs crystals which are almost completely composed of $\{111\}$ surfaces, indicating enhanced lattice distortion in the facets as a result of elastic relaxation of the thermal strain.

The GaAs parallel strain dependence on GaAs/Ge crystal height is plotted in Figure 10(e). The height values correspond to the maximum ones measured along the (001) direction, which are different from the nominal ones, as stated in Section III B. These results were compared with FEM simulations of the elastic relaxation of the thermal strain performed for realistic geometries based on the dimensions extracted from SEM cross section images. The used boundary conditions and initial parameters are the same as those in Sec. III C 1. The strain relaxation maps of the GaAs/Ge crystals of the studied heights grown on $15-\mu \mathrm{m}$-wide Si pillars are presented in Figure 10(f). The good agreement between the theoretical curve obtained from the FEM simulations and the HRXRD experimental data can be observed in Fig. 10(e).

\section{SUMMARY}

In summary, GaAs/Ge crystals grown on patterned $\mathrm{Si}$ substrates show a truncated pyramidal morphology dominated by $\{111\}$ planes. The crystals apex is formed by $\{137\}$ lateral facets symmetrically arranged around the [110] direction and two main top surfaces: one parallel to the offcut substrate and the other corresponding to the real GaAs (001) crystallographic plane. We explained these morphologies by a combination of the anisotropic growth kinetics of GaAs and growth on vicinal substrates. The lower density of defects found in the GaAs/Ge crystals grown on 2- $\mu \mathrm{m}$-wide $\mathrm{Si}(001)$ pillars compared with the one found for the $15-\mu \mathrm{m}$ wide ones suggests that further miniaturization, even down to the nanometer scale, may be necessary in order to obtain defect free GaAs on Si substrates. Nanoheteroepitaxy theory predicts that direct growth of GaAs and $\mathrm{Ge}$ on $\mathrm{Si}$ (both systems with $\sim 4 \%$ misfit) should support a coherent interface provided the surface size is below the critical nucleation island diameter of $40 \mathrm{~nm} .{ }^{55}$ Scaling down the pillar size to the nanometer has been recently reported for the growth of Ge in compliant Si nano-substrates. ${ }^{56}$ Even though the conceptual interest of this approach is out of discussion, such small sizes still complicate to a large extent the technological steps needed for potential applications.

A tentative option would consist in the growth of thicker, faceted Ge pillars before the GaAs growth. Previous works demonstrated defect free Ge monolithically integrated on $\mathrm{Si}^{36}$ After 3-4 $\mu \mathrm{m}$, the Ge crystals grown on $4 \mu \mathrm{m}^{2}$ Si pillars have expelled out all the dislocations by both ART and faceting. Further growth of GaAs layers below the critical thickness on such defect-free Ge crystals should provide defect free materials.

Finally, several conclusions could be drawn regarding the influence of the Si pillar aspect ratio and the substrate orientation on the relaxation process of the thermal strain. A complete elastic release of the thermal strain is found in 
GaAs crystals grown on $5 \times 5 \mu \mathrm{m}^{2}$ Si pillars compared with the GaAs layers grown on planar substrates. Further increments of the crystals aspect ratio lead to a complete elastic release of the thermal strain accumulated in the GaAs. Additionally, it was found that the lattice tilt of $\mathrm{GaAs} / \mathrm{Ge}$ layers along the offcut is modified under the action of an asymmetric stress, underlining the potential of the 3Dheteroepitaxy technique in order to tailor the crystallographic properties of heteroepitaxial layers.

\section{ACKNOWLEDGMENTS}

Financial support by the Sinergia project NOVIPIX and the DACH program (200021L_153558) of the Swiss National Science Foundation is greatly appreciated. Part of this work was supported by ETH Research Grant ETH-20 11-2. We acknowledge K. Mattenberger for technical support and B. Batlogg and E. Gini for valuable scientific discussions; P. Niedermann is acknowledged for the $\mathrm{Si}$ patterned substrates fabrication. FIRST center for micro- and nanoscience and EMEZ electron microscopy center at ETH Zürich for making available its infrastructures and Pilegrowth Tech. Srl for providing their technical expertise. We want to acknowledge to CEITEC open access project LM2011020 as well, funded by Czech Ministry of Education and one author wishes to acknowledge also the projects Research4Industry (CZ.1.07/2.4.00/17.0006), NANOEEE2.3.20.0027 and ED1.1.00/02.0068.

${ }^{1}$ S. Lourdudoss, Curr. Opin. Solid State Mater. Sci. 16, $91-99$ (2012).

${ }^{2}$ P. M. Petroff, J. Vac. Sci. Technol., B 4, 874 (1986).

${ }^{3}$ Y. Li, G. Salviati, M. M. G. Bongers, L. Lazzarini, L. Nasi, and L. J. Giling, J. Cryst. Growth 163, 195 (1996).

${ }^{4}$ M. Akiyama, Y. Kawarada, and K. Kaminishi, J. Cryst. Growth 68, 21-26 (1984).

${ }^{5}$ G. Brammertz, Y. Mols, S. Degroote, V. Motsnyi, M. Leys, G. Borghs, and M. Caymax, J. Appl. Phys. 99, 093514 (2006).

${ }^{6}$ L. Knuuttila, A. Lankinen, J. Likonen, H. Lipsanen, X. Lu, P. McNally, J. Riikonen, and T. Tuomi, Jpn. J. Appl. Phys., Part 1 44, 7777 (2005).

${ }^{7}$ K. Nozawa and Y. Horikoshi, Jpn. J. Appl. Phys., Part 2 29, L540-L543 (1990).

${ }^{8}$ R. Fischer, N. Chand, W. Kopp, H. Morkog, L. P. Erickson, and R. Youngman, Appl. Phys. Lett. 47, 397 (1985).

${ }^{9}$ Y. González, L. González, and F. Briones, Jpn. J. Appl. Phys., Part 2 31, L816-L819 (1992).

${ }^{10}$ J. M. Olson, M. M. Al-Jassim, A. Kibbler, and K. M. Jone, J. Cryst. Growth 77, 515 (1986).

${ }^{11}$ Y. Komatsu, K. Hosotani, T. Fuyuki, and H. Matsunami, Jpn. J. Appl. Phys., Part 1 36, 5425 (1997).

${ }^{12}$ Y. Takagi, H. Yonezu, T. Kawai, K. Hayashida, K. Samonji, N. Ohshima, and K. Pak, J. Cryst. Growth 150, 677 (1995).

${ }^{13}$ T. Tsuji, H. Yonezu, and N. Ohshima, J. Vac. Sci. Technol., B 22, 1428 (2004).

${ }^{14}$ K. Nozawa and Y. Horikoshi, Jpn. J. Appl. Phys., Part 2 30, L668 (1991).

${ }^{15}$ S. M. Ting and E. A. Fitzgerald, J. Appl. Phys. 87, 2618 (2000).

${ }^{16}$ J. A. Carlin, S. A. Ringel, E. A. Fitzgerald, M. Bulsara, and B. M. Keyes, Appl. Phys. Lett. 76, 1884 (2000).

${ }^{17}$ R. Ginige, B. Corbett, M. Modreanu, C. Barrett, J. Hilgarth, G. Isella, D. Chrastina, and H. von Känel, Semicond. Sci. Technol. 21(6), 775-780 (2006).

${ }^{18}$ S. Bietti, C. Somaschini, S. Sanguinetti, N. Koguchi, G. Isella, and D. Chrastina, Appl. Phys. Lett. 95(24), 241102 (2009).

${ }^{19}$ H. P. Singh, Acta Cryst. A 24, 469 (1968).

${ }^{20}$ J. S. Blakemore, J. Appl. Phys. 53, R123 (1982).

${ }^{21}$ Y. Okada and Y. Tokumaru, J. Appl. Phys. 56, 314 (1984).

${ }^{22}$ C. V. Falub, H. von Känel, F. Isa, R. Bergamaschini, A. Marzegalli, D. Chrastina, G. Isella, E. Müller, P. Niedermann, and L. Miglio, Science 335, 1330 (2012).
${ }^{23}$ J.-S. Park, J. Bai, M. Curtin, B. Adekore, M. Carroll, and A. Lochtefeld, Appl. Phys. Lett. 90, 052113 (2007).

${ }^{24}$ E. A. Fitzgerald and N. Chand, J. Electron. Mater. 20, 839 (1991).

${ }^{25}$ C. V. Falub, T. Kreiliger, F. Isa, A. G. Taboada, M. Meduňa, F. Pezzoli, R. Bergamaschini, A. Marzegalli, E. Müller, D. Chrastina, G. Isella, A. Neels, P. Niedermann, A. Dommann, L. Miglio, and H. von Känel, Thin Solid Films 557, 42-49 (2014).

${ }^{26}$ A. G. Taboada, T. Kreiliger, C. V. Falub, F. Isa, M. Salvalaglio, L. Wewior, D. Fuster, M. Richter, E. Uccelli, P. Niedermann, A. Neels, F. Mancarella, B. Alén, L. Miglio, A. Dommann, G. Isella, and H. von Känel, Appl. Phys. Lett. 104(2), 022112 (2014).

${ }^{27}$ M. Richter, E. Uccelli, A. G. Taboada, D. Caimi, N. Daix, M. Sousa, C. Marchiori, H. Siegwart, C. V. Falub, H. von Känel, F. Isa, G. Isella, A. Pezous, A. Dommann, P. Niedermann, and J. Fompeyrine, J. Cryst. Growth 378, 109-112 (2013).

${ }^{28}$ S. Bietti, A. Scaccabarozzi, C. Frigeri, M. Bollani, E. Bonera, C. V. Falub, H. von Känel, L. Miglio, and S. Sanguinetti, Appl. Phys. Lett. 103(26), 262106 (2013).

${ }^{29}$ A. Jung, A. G. Taboada, W. Stumpf, T. Kreiliger, F. Isa, G. Isella, E. Barthazy Meier, and H. von Känel, J. Appl. Phys. 118, 075701 (2015).

${ }^{30}$ F. Laermer and A. Schilp, U.S. patent 5501893 (26 Mar 1996).

${ }^{31}$ C. Rosenblad, H. von Känel, M. Kummer, A. Domman, and E. Muller, Appl. Phys. Lett. 76, 427 (2000).

${ }^{32}$ J. T. Robinson, A. Rastelli, O. Schmidt, and O. D. Dubon, Nanotechnology 20, 085708 (2009).

${ }^{33}$ R. Bergamaschini, F. Isa, C. V. Falub, P. Niedermann, E. Müller, G. Isella, H. von Känel, and L. Miglio, Surf. Sci. Rep. 68, 390 (2013).

${ }^{34}$ S. Marchionna, A. Virtuani, M. Acciarri, G. Isella, and H. von Kaenel, Mater. Sci. Semicond. Process. 9, 802 (2006).

${ }^{35}$ L. D. Landau and E. M. Lifshitz, Theory of Elasticity (Pergamo Press, Oxford, 1970), Vol. 7.

${ }^{36}$ C. V. Falub, M. Meduňa, D. Chrastina, F. Isa, A. Marzegalli, T. Kreiliger, A. G. Taboada, G. Isella, L. Miglio, A. Dommann, and H. von Känel, Sci. Rep. 3, 2276 (2013).

${ }^{37}$ S. C. Lee, D. L. Huffaker, and S. R. J. Brueck, Appl. Phys. Lett. 92, 023103 (2008).

${ }^{38}$ X. Q. Shen, M. Tanaka, K. Wada, and T. Nishinaga, J. Cryst. Growth 135(1), 85-96 (1994).

${ }^{39}$ D. J. Chadi, Phys. Rev. Lett. 43, 43 (1979).

${ }^{40}$ F. Riesz, J. Vac. Sci. Technol., A 14, 425 (1996).

${ }^{41}$ H. Nagai, J. Appl. Phys. 45, 3789 (1974).

${ }^{42}$ G. H. Olsen and R. T. Smith, Phys. Status Solidi A 31, 739 (1975).

${ }^{43}$ L. J. Schowalter, E. L. Hall, N. Lewis, and S. Hashimoto, Thin Solid Films 184, 437-445 (1990).

${ }^{44}$ J. E. Ayers, S. K. Ghandhi, and L. J. Schowalter, J. Cryst. Growth 113, 430 (1991).

${ }^{45}$ D. Colombo, E. Grilli, M. Guzzi, S. Sanguinetti, S. Marchionna, M. Bonfanti, A. Fedorov, H. von Känel, G. Isella, and E. Müller, J. Appl. Phys. 101, 103519 (2007).

${ }^{46}$ V. K. Yang, M. Groenert, C. W. Leitz, A. J. Pitera, M. T. Currie, and E. A. Fitgerald, J. Appl. Phys. 93, 3859 (2003).

${ }^{47}$ J. E. Ayers, J. Cryst. Growth 135, 71 (1994).

${ }^{48}$ M. A. Krivoglaz, X-ray and Neutron Diffraction of Nonideal Crystals (Springer, Berlin, 1996).

${ }^{49}$ V. M. Kaganer, B. Köhler, M. Schmidbauer, R. Opitz, and B. Jenichen, Phys. Rev. B 55, 1793 (1997).

${ }^{50}$ V. M. Kaganer and K. K. Sabelfeld, Phys. Rev. B 80, 184105 (2009).

${ }^{51}$ L. Tapfer, J. R. Martinez, and K. Ploog, Semicond. Sci. Technol. 4, 617-621 (1989).

${ }^{52}$ D. I. Westwood, D. A. Woolf, S. A. Clark, and R. H. Williams, Mater. Sci. Eng., B 9, 151-155 (1991).

${ }^{53}$ M. Tachikawa and H. Mori, Appl. Phys. Lett. 56, 2225 (1990).

${ }^{54}$ G. Capellini, M. De Seta, P. Zaumseil, G. Kozlowski, and T. Schroeder, J. Appl. Phys. 111, 073518 (2012).

${ }^{55}$ D. Zubia, S. H. Zaidi, S. D. Hersee, and S. R. J. Brueck, J. Vac. Sci. Technol., B 18, 3514 (2000).

${ }^{56}$ P. Zaumseil, G. Kozlowski, Y. Yamamoto, J. Bauer, M. A. Schubert, T. U. Schülli, B. Tillack, and T. Schroeder, J. Appl. Phys. 112, 043506 (2012). 